\title{
COUPLED-CAVITY STRUCTURES IN PHOTONIC CRYSTALS
}

\author{
MEHMET BAYINDIR \\ EKMEL OZBAY
}

Department of Physics, Bilkent University, Turkey 


\section{$>$ Motivations}

$>$ Underlying Physics

$>$ Investigation of coupled-cavity structures: FDTD, TMM, experiment, and tight-binding approximation

$>$ Localized cavity modes

$>$ Eigenmose splitting

$>$ Photonic molecules

$>$ Observation of a new type of waveguiding mechanism:

Coupled-cavity waveguides (CCWs)

$>$ Possible Applications

$>$ Waveguides, waveguide bends, splitters, switches

$>$ WDM: adding or dropping a selective wavelength or band

$>$ Strong enhancement of the spontaneous emission

$>$ Increasing efficiency of nonlinear processes

$>$ Dispersion compensators

$>$ Summary 


\section{PHOTONIC INTEGRATED CIRCUIT}

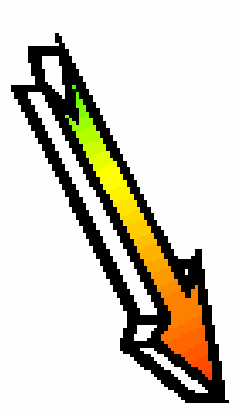

Monolithic wavelength converter

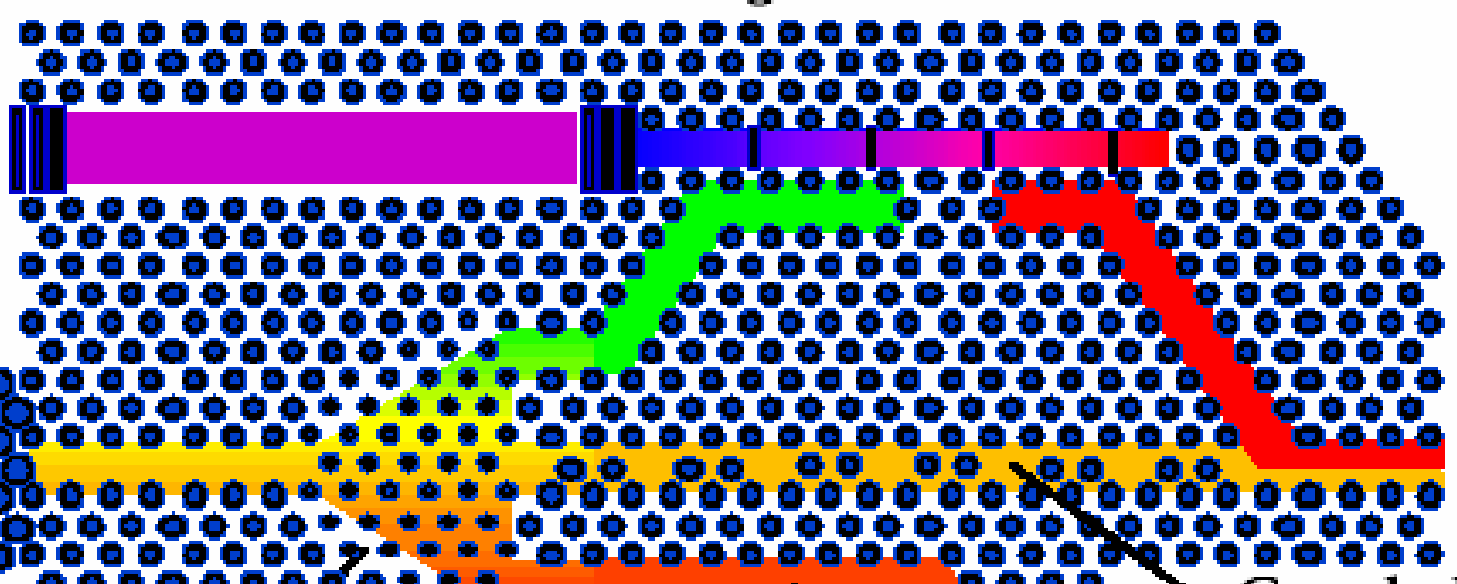

Fibre coupler (in)

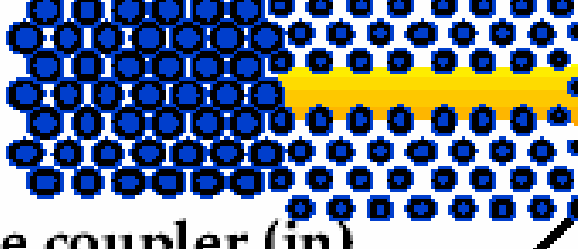
\%o

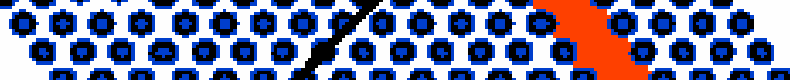

Highly dispersive element ("superprism")

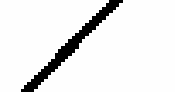$$
\text { element ("superprism") }
$$

Channel waveguide
Coupled cavity waveguide

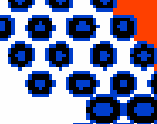

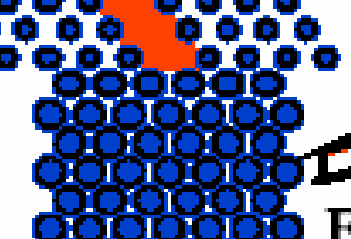
Fibre coupler (out)

[from Krauss' paper] 

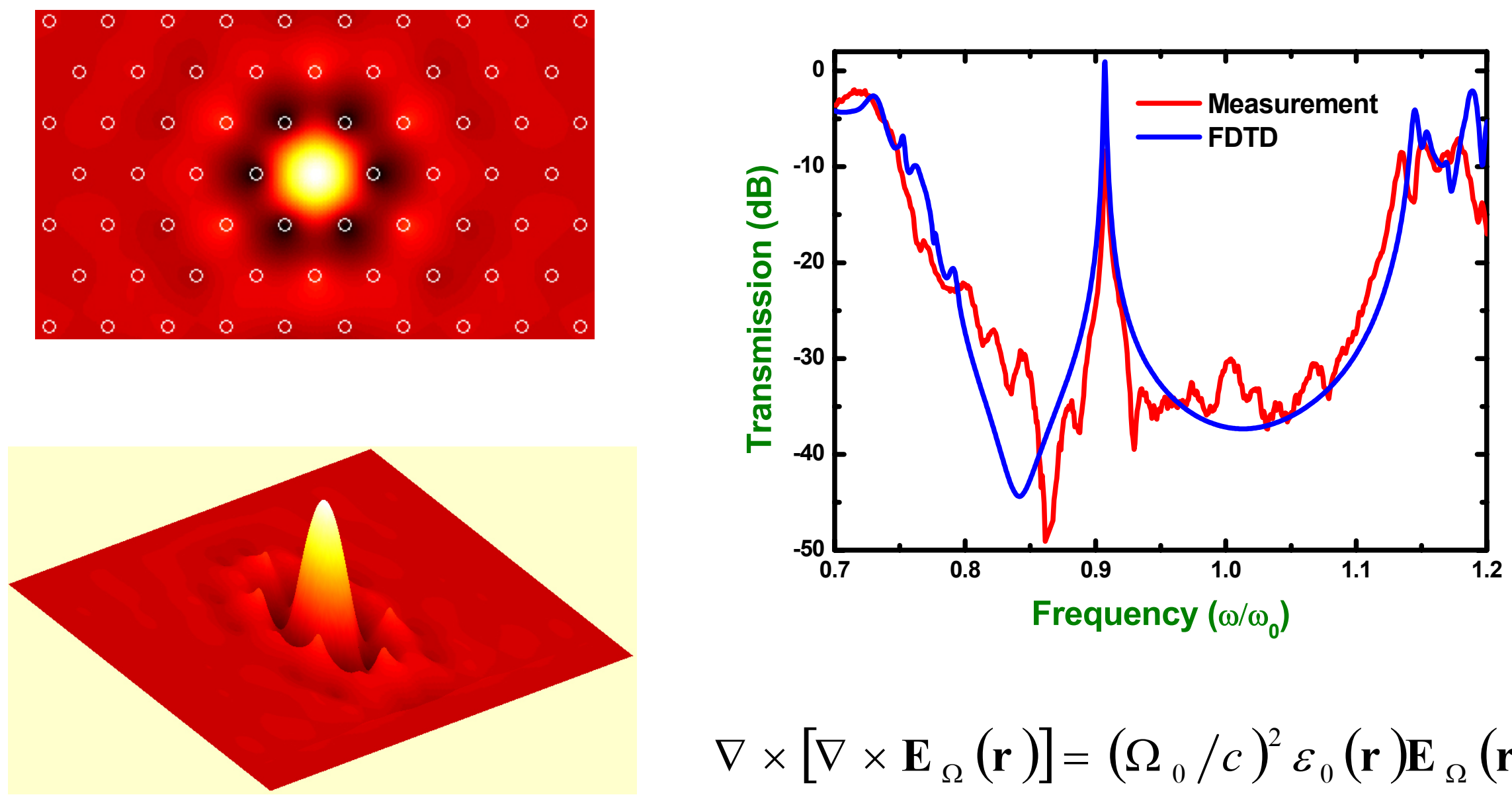

$$
\nabla \times\left[\nabla \times \mathbf{E}_{\Omega}(\mathbf{r})\right]=\left(\Omega_{0} / c\right)^{2} \varepsilon_{0}(\mathbf{r}) \mathbf{E}_{\Omega}(\mathbf{r})
$$

Observation of strongly localized cavity modes within the photonic band gap analogous to acceptor impurity state in semiconductors 


\section{EIGENMODE SPLITTING}

$$
\mathbf{E}_{\omega}(\mathbf{r})=\mathrm{AE}_{\Omega}(\mathbf{r})+\mathrm{BE}_{\Omega}(\mathbf{r}-\Lambda) \quad \begin{aligned}
& \text { Linear combination of the individual } \\
& \text { evanescent cavity modes }
\end{aligned}
$$

$$
\nabla \times\left[\nabla \times \mathbf{E}_{\omega}(\mathbf{r})\right]=(\omega / \mathrm{c})^{2} \varepsilon_{0}(\mathbf{r}) \mathbf{E}_{\omega}(\mathbf{r})
$$

$$
\omega_{1,2}=\Omega \sqrt{\frac{1 \pm \beta}{1 \pm \alpha}}
$$

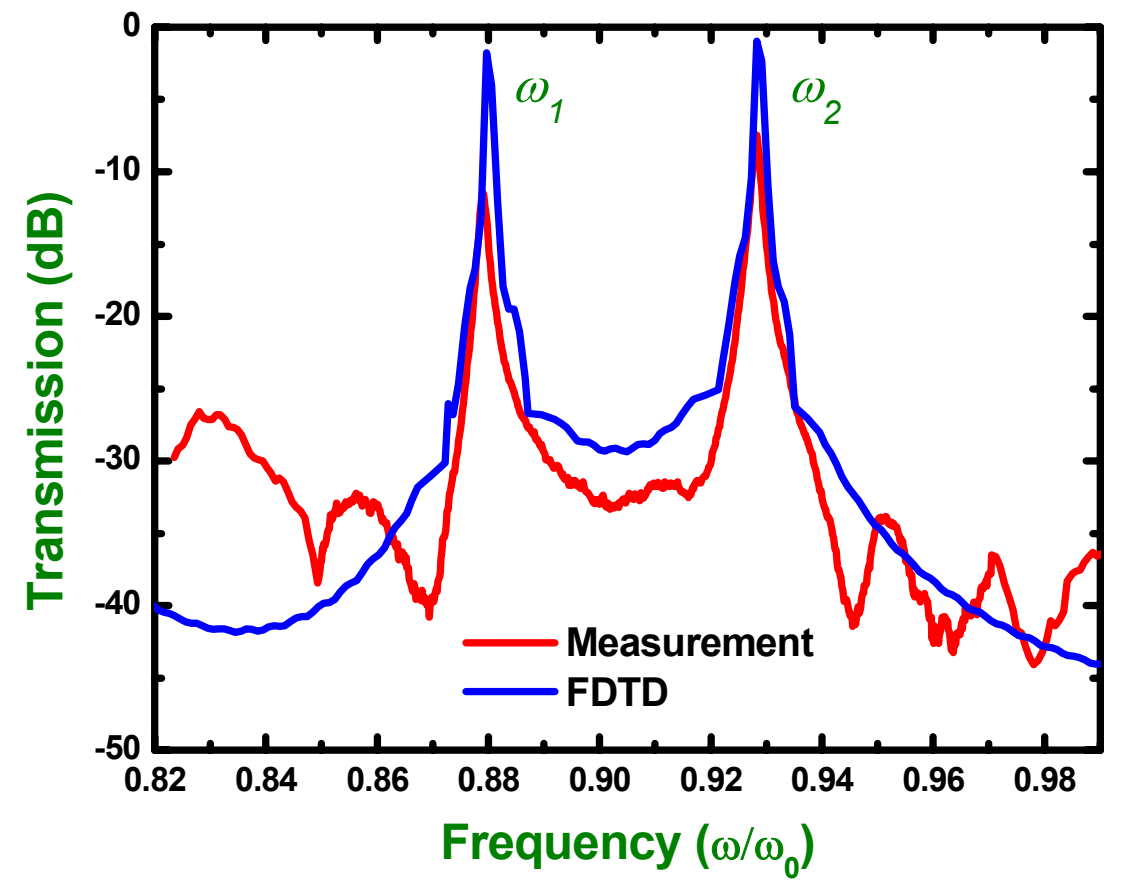

$$
E_{\mathrm{a}}=\frac{E_{\mathrm{Q}}(\mathrm{r})-E_{\mathrm{Q}}(\mathrm{r}-\Lambda)}{\sqrt{2}}
$$

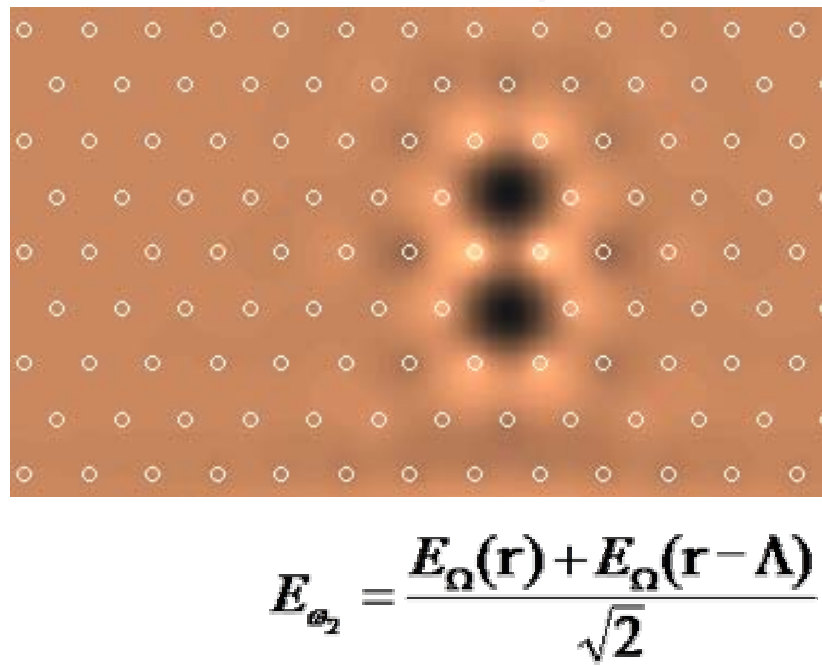

Formation of bondling and antibonding photonic modes 
$E_{\mathrm{T}_{\mathrm{i}}}=\frac{E_{\mathrm{n}}(\mathrm{I})-\sqrt{2} E_{\mathrm{n}}(\mathrm{r}-\Lambda)+E_{\mathrm{n}}(\mathrm{r}-2 \Lambda)}{2} \quad E_{\mathrm{T}_{2}}=\frac{E_{\mathrm{n}}(\mathrm{r})-E_{\mathrm{n}}(\mathrm{r}-2 \Lambda)}{\sqrt{2}} \quad E_{\mathrm{T}_{3}}=\frac{E_{\mathrm{n}}(\mathrm{r})+\sqrt{2} E_{\mathrm{n}}(\mathrm{\textrm {T }}-\Lambda)+E_{\mathrm{n}}(\mathrm{r}-2 \Lambda)}{2}$
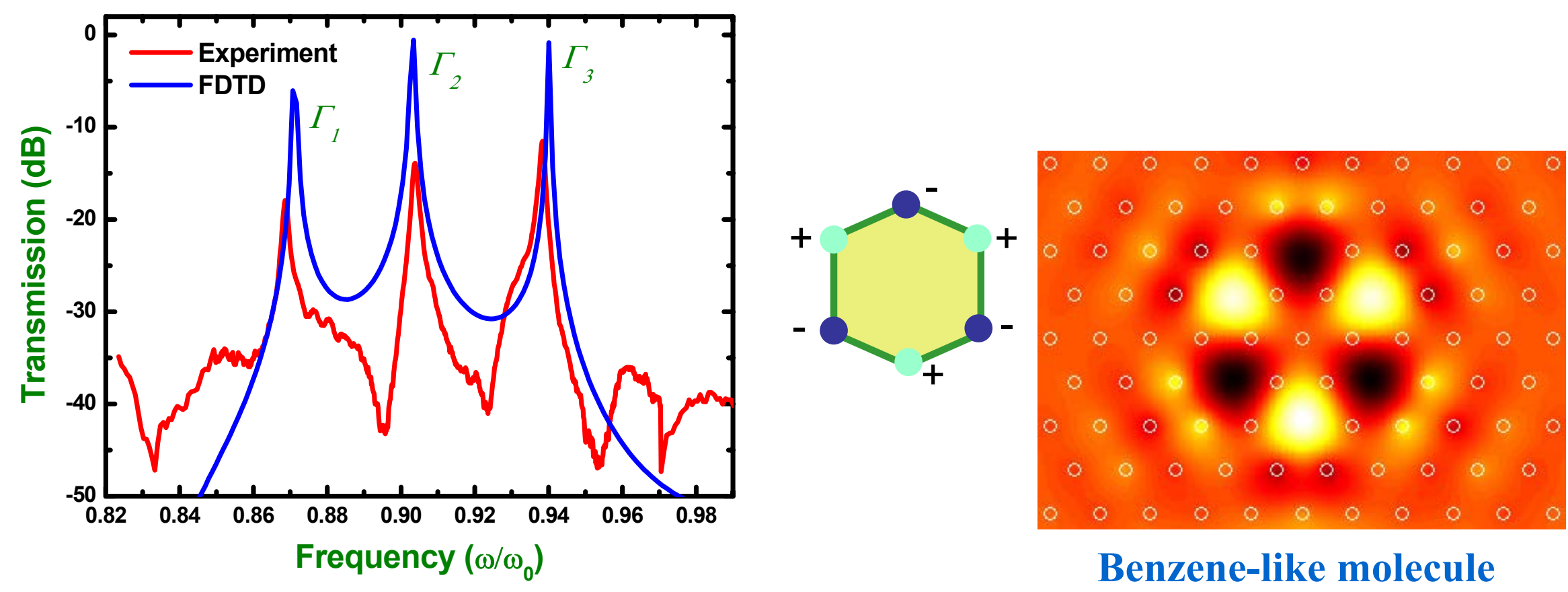

Benzene-like molecule 


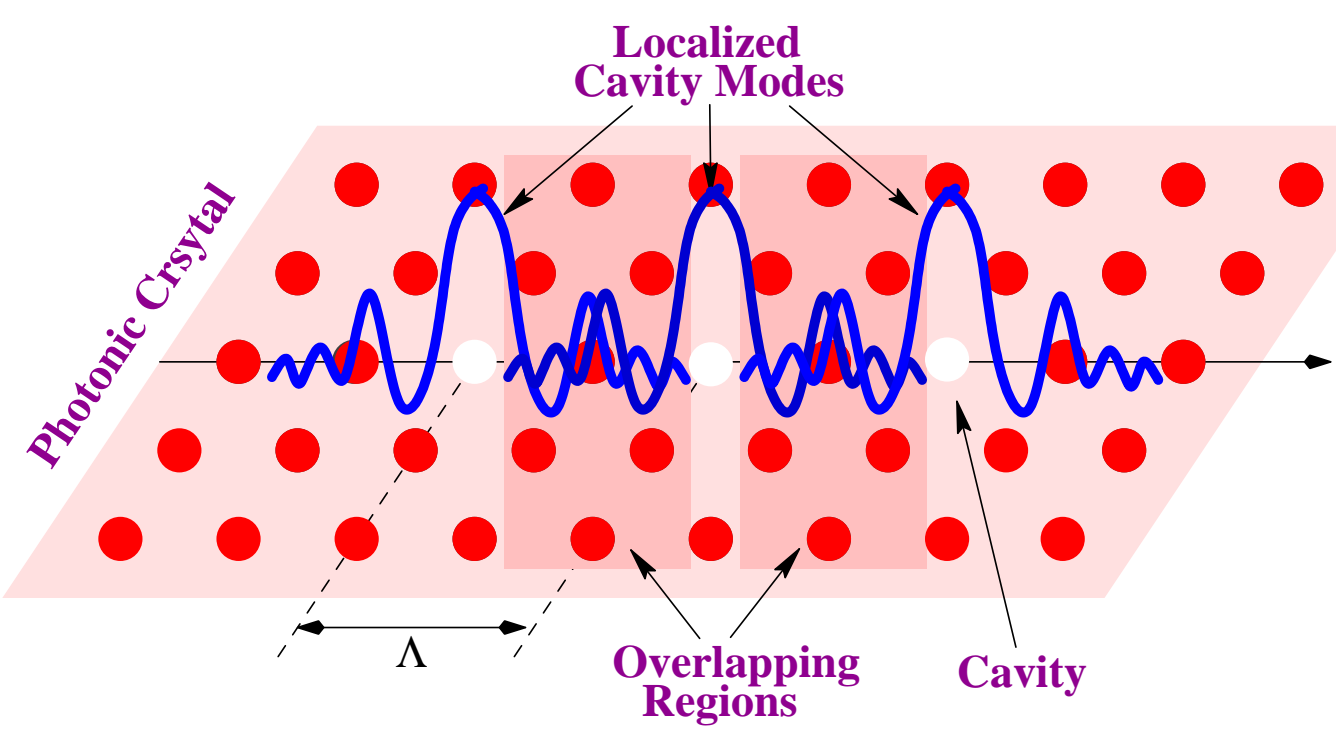

$$
\begin{aligned}
& \nabla \times[\nabla \times \mathbf{E}(\mathbf{r})]=(\omega / \mathrm{c})^{2} \varepsilon_{0}(\mathbf{r}) \mathbf{E}(\mathbf{r}) \\
& \mathbf{E}(\mathbf{r})=E_{0} \sum_{n} e^{-i n k \Lambda} \mathbf{E}_{\Omega}(\mathbf{r}-n \Lambda) \\
& \omega(k)=\Omega(1+\kappa \cos (k \Lambda)) \\
& v_{g}(k)=\nabla_{k} \omega(k)=-\Omega \Lambda \kappa \sin (k \Lambda)
\end{aligned}
$$

$\checkmark$ highly localized

$\checkmark$ weakly interacting cavity modes

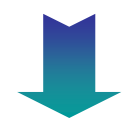

$$
\tau_{p}(k)=L / v_{g}(k)-2 \pi L / c
$$

\section{Tight-binding approximation}

Dispersion relation, group velocity, and photon lifetime depend only a single tight-binding parameter $\kappa$ that can be directly determined from experiments 


\section{PROPAGATION OF PHOTONS BY HOPPING}
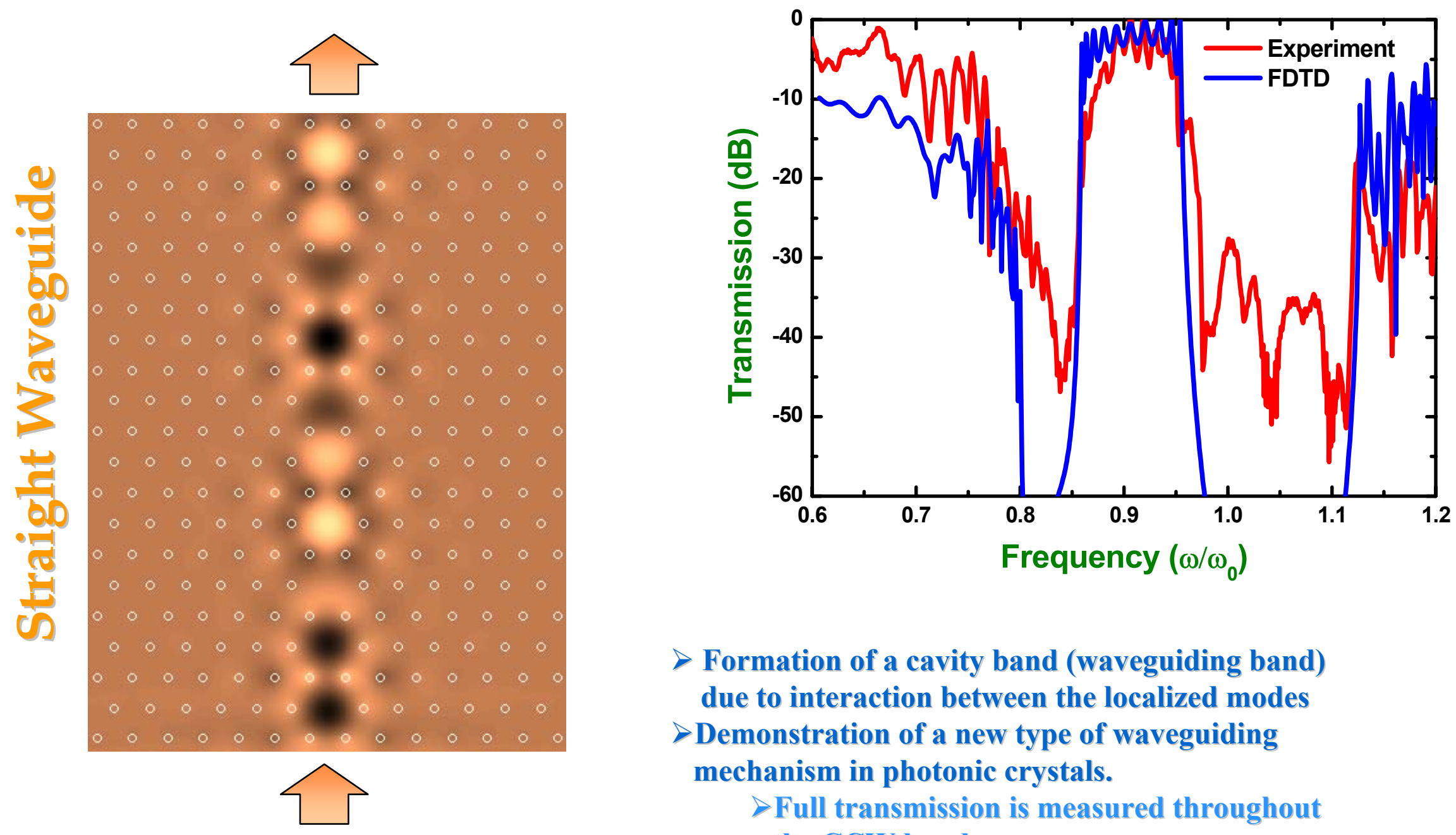

Formation of a cavity band (waveguiding band) due to interaction between the localized modes $>$ Demonstration of a new type of waveguiding mechanism in photonic crystals.

$>$ Full transmission is measured throughout the CCW band

$>$ Very sharp band edges can be used for switching applications

Bayindir, et al., Phys. Rev. B 61, R11855 (2000); JQE (in press, 2002) 
DISPERSION RELATION, GROUP VELOCITY, PHOTON LIFETIME:

MEASUREMENTS AND CALCULATIONS
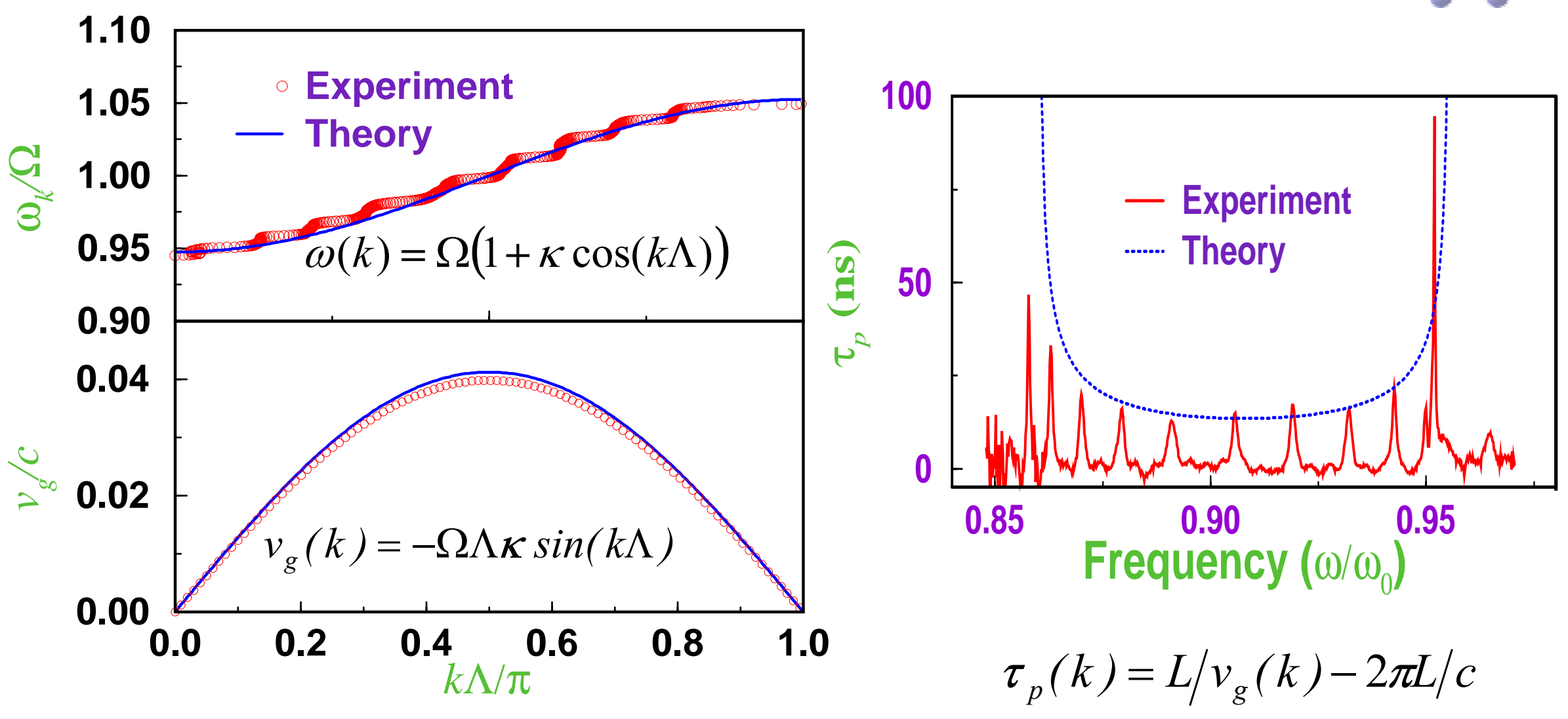

$$
\begin{gathered}
v_{g} \rightarrow 0 \\
\tau_{p} \rightarrow \infty
\end{gathered}
$$

at the $\mathrm{CCW}$ band edges

Bayindir and Ozbay, Phys. Rev. B 62, R2247 (2000) 
BENDING OF EM WAVES ALONG ARBITRARY PATH

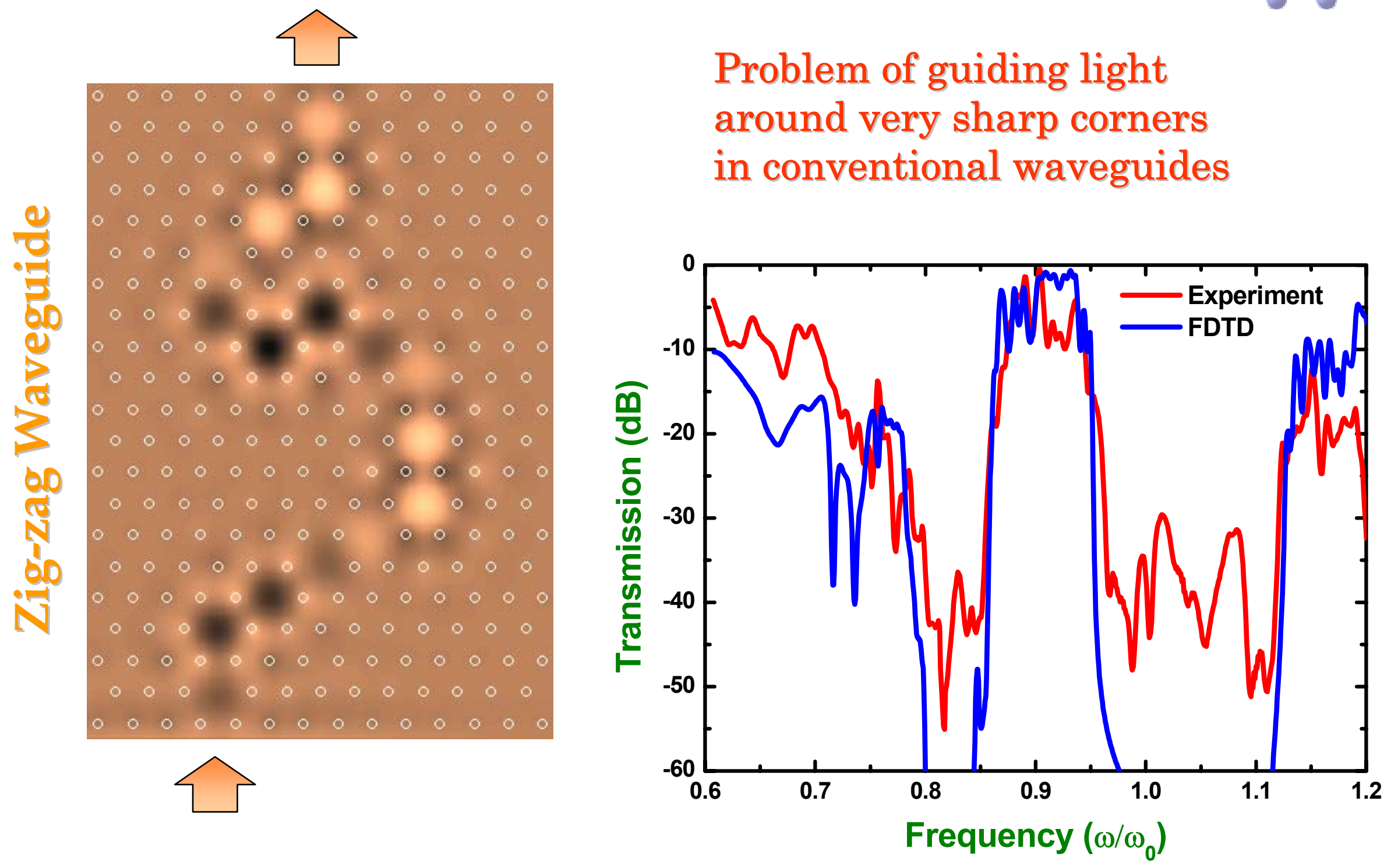

$>$ Possibility of constructing lossless and reflectionless bends in optical circuits

Bayindir, et al., Phys. Rev. B 61, R11855 (2000) 


\section{1}
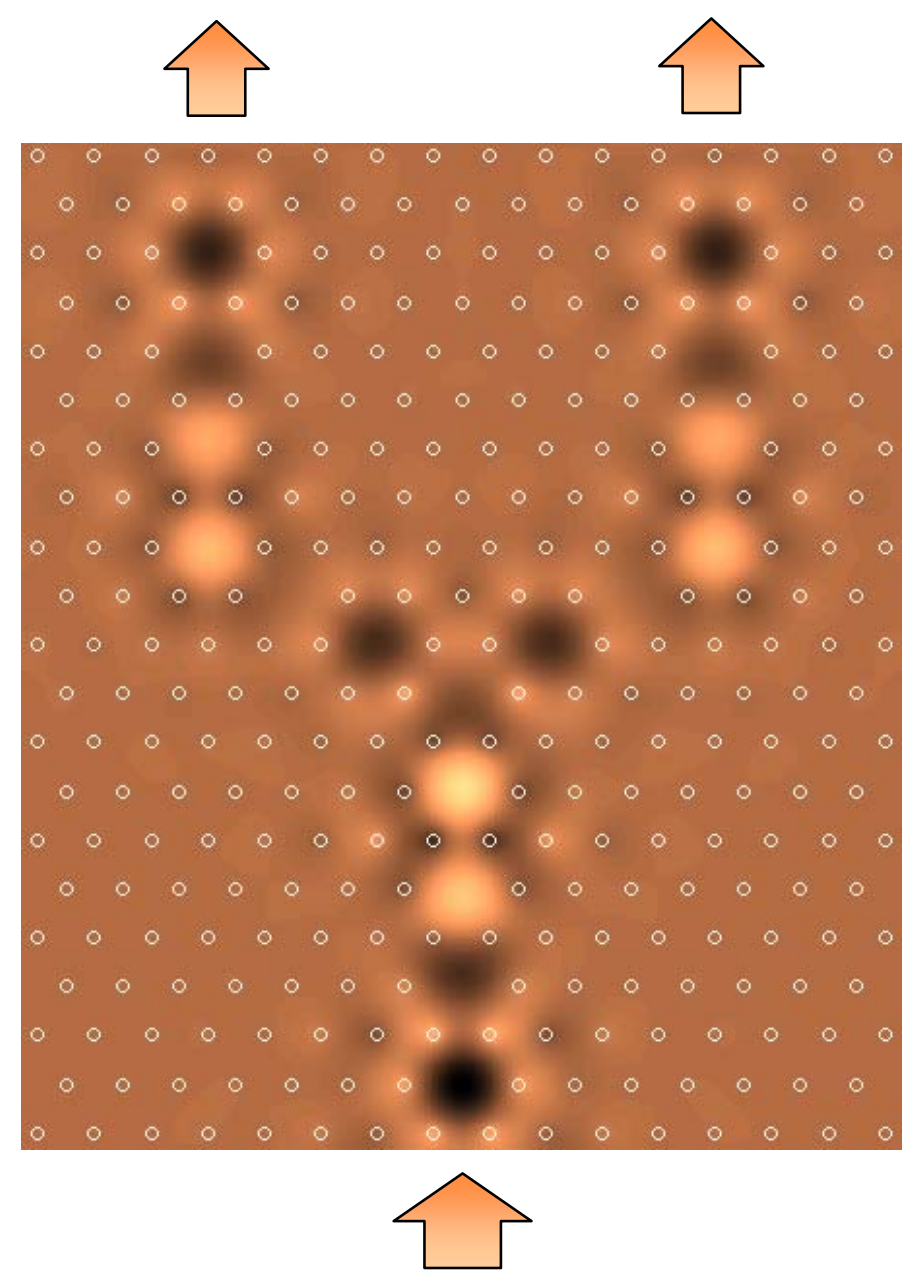
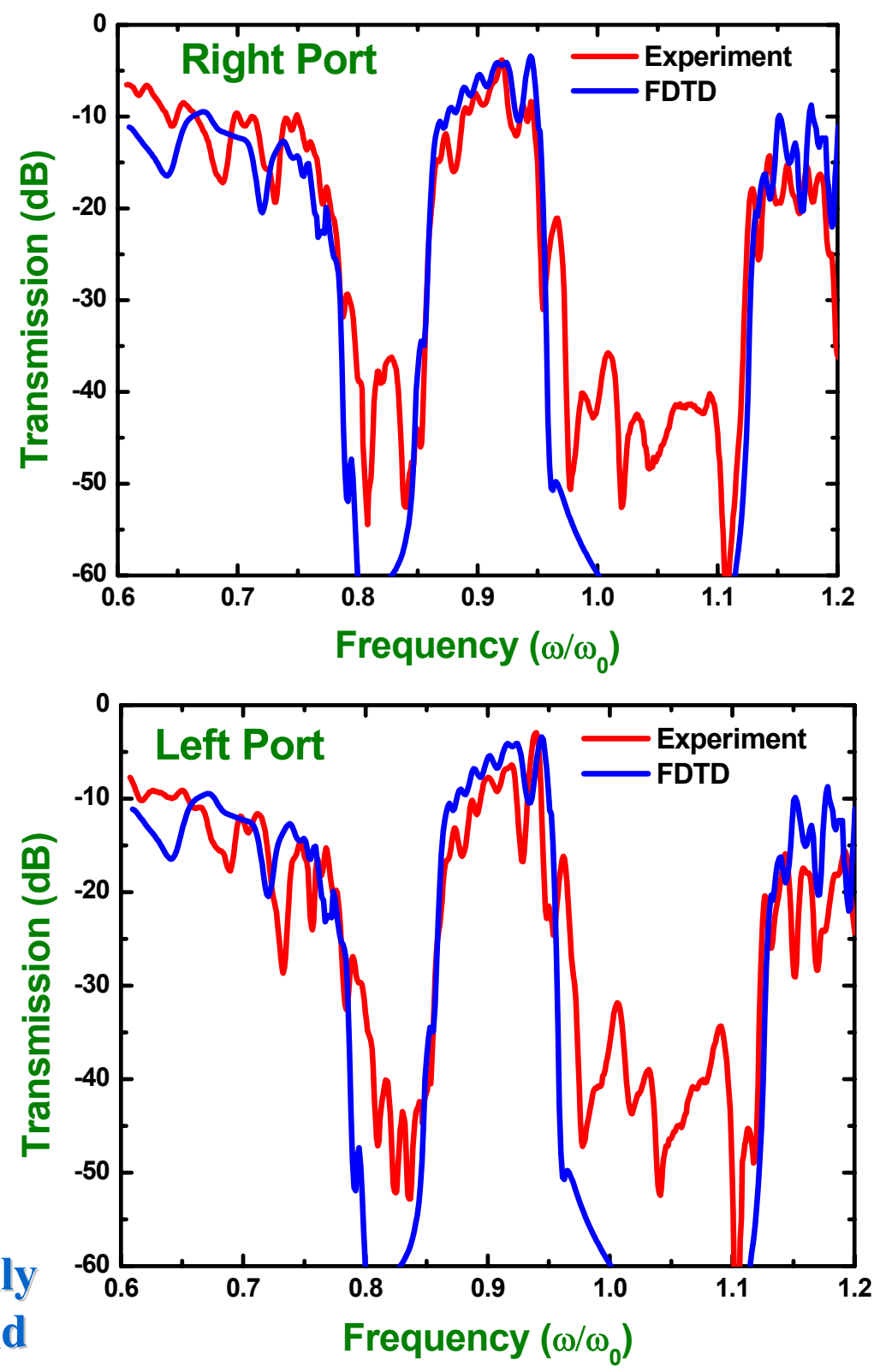

Bayindir, et al., Appl. Phys. Lett. 77, 3902 (2000), JQE (in press) 

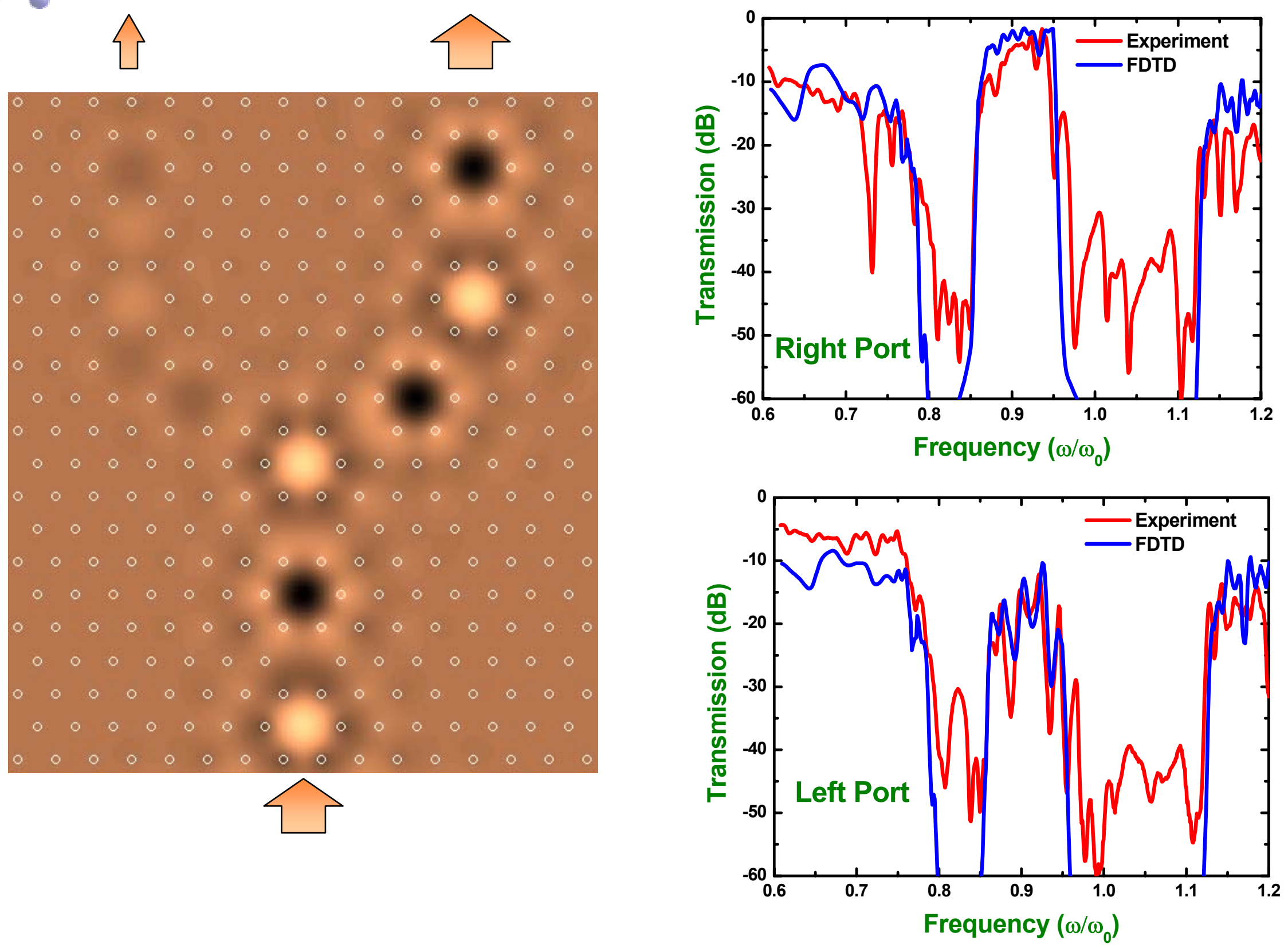


\section{WDM APPLICATIONS}

Single wavelength dropping

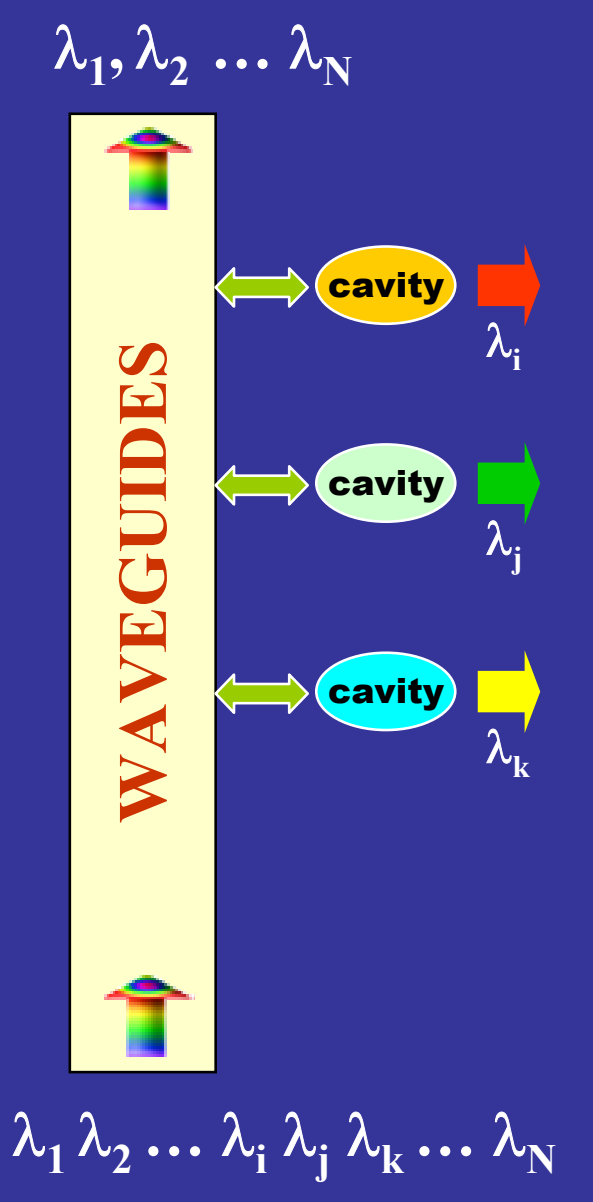

Band dropping

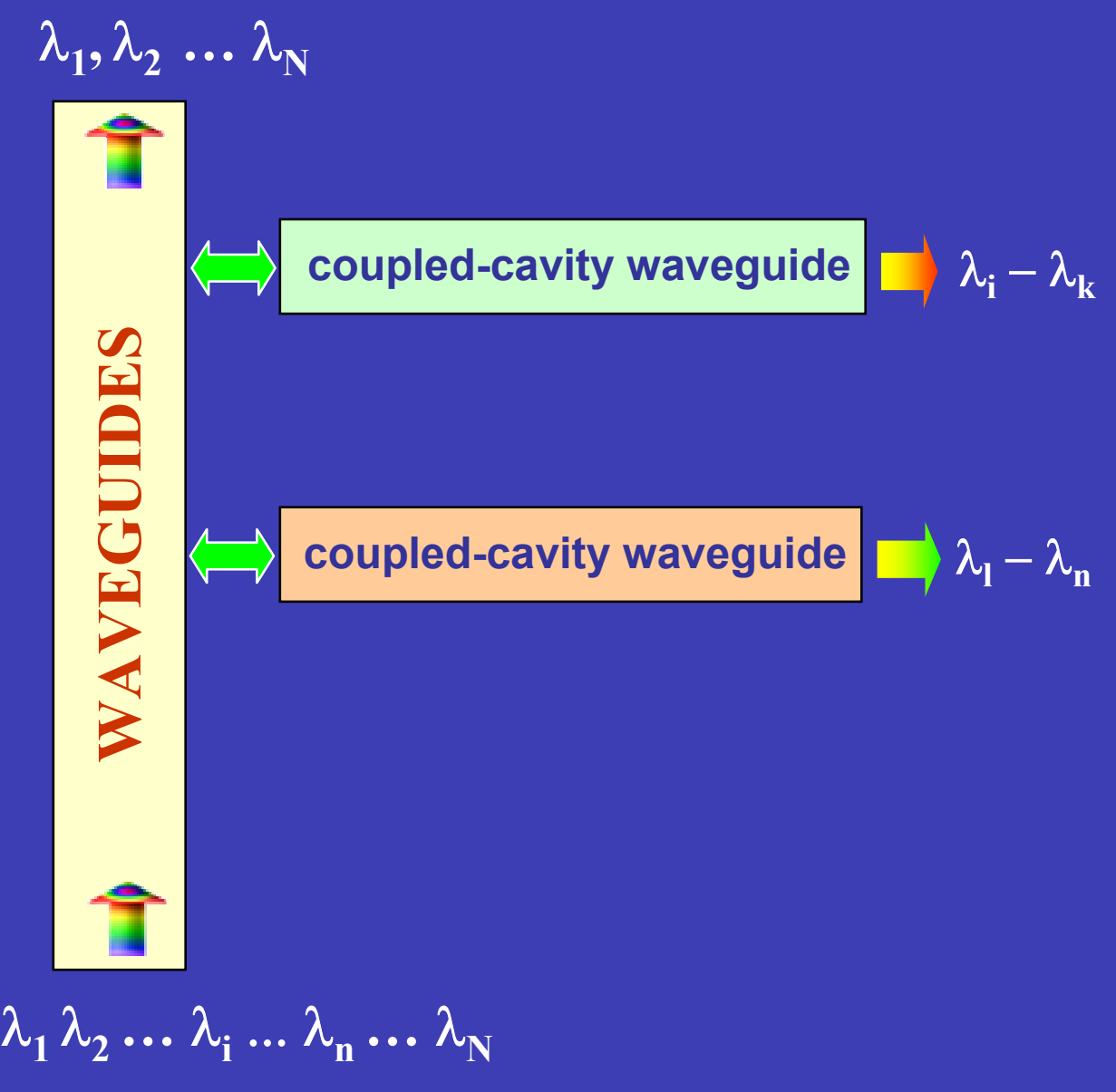


DROPPING OF A SELECTIVE FREQUENCY FROM PW
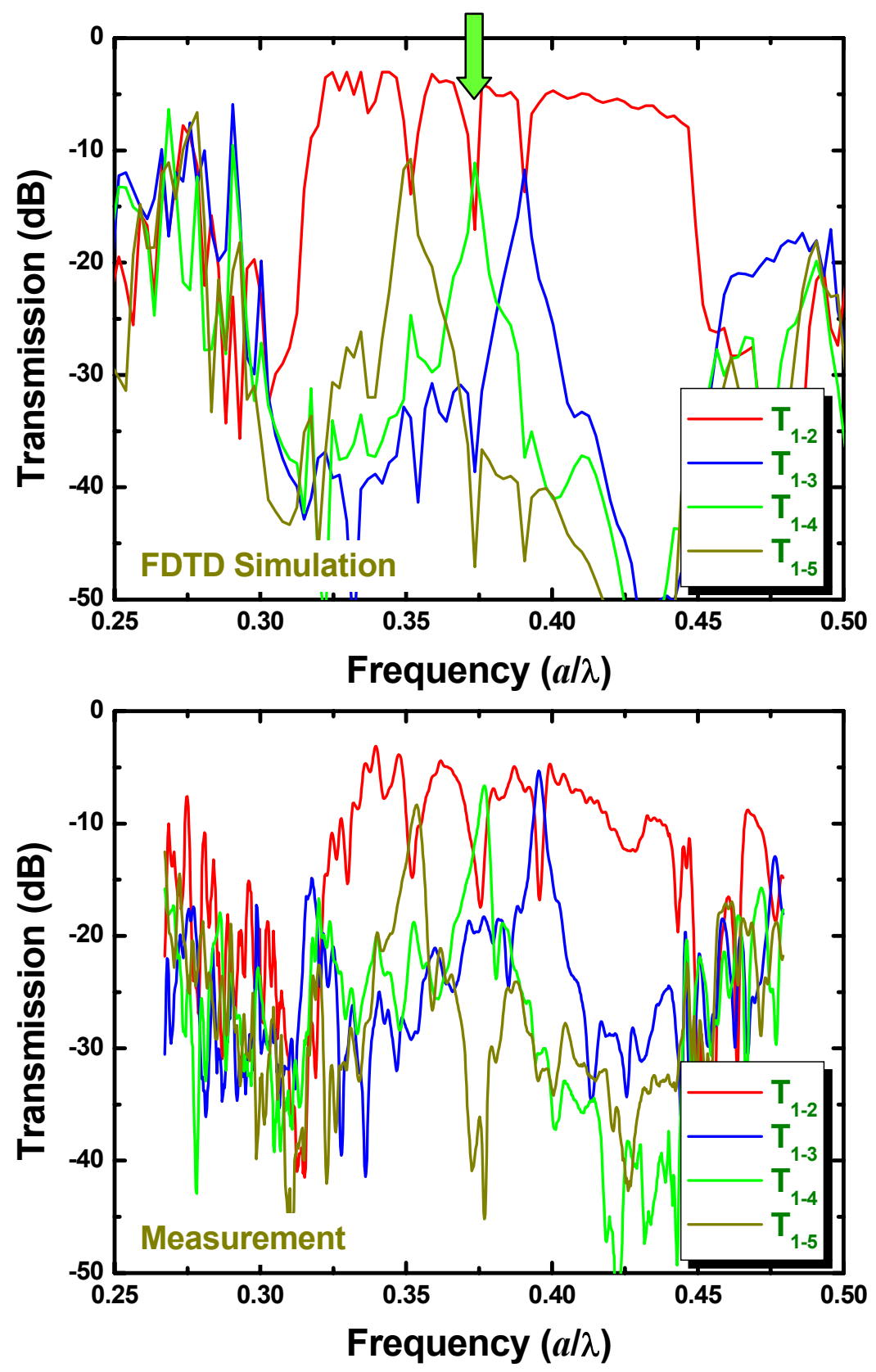

(2)

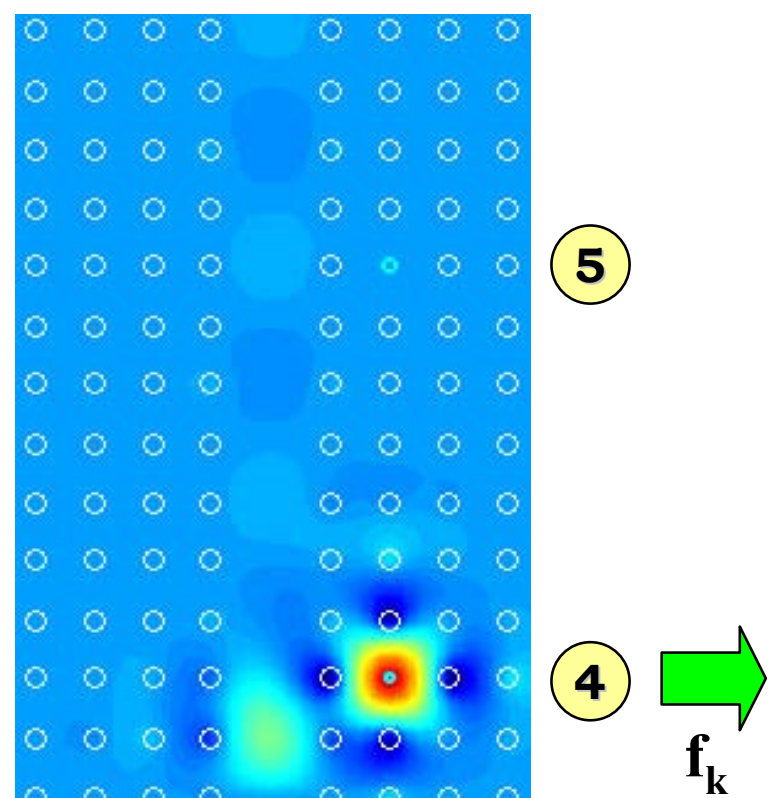

$\begin{array}{llllllll}0 & 0 & 0 & 0 & 0 & 0 & 0 & 0 \\ 0 & 0 & 0 & 0 & 0 & 0 & 0 & 0 \\ 0 & 0 & 0 & 0 & 0 & 0 & 0 & 0 \\ 0 & 0 & 0 & 0 & 0 & 0 & 0 & 0 \\ 0 & 0 & 0 & 0 & 0 & & 0 & 0 \\ 0 & 0 & 0 & 0 & 0 & 0 & 0 & 0 \\ 0 & 0 & 0 & 0 & 0 & 0 & 0 & 0 \\ 0 & 0 & 0 & 0 & 0 & 0 & 0 & 0 \\ 0 & 0 & 0 & 0 & 0 & 0 & 0 & 0 \\ 0 & 0 & 0 & 0 & 0 & 0 & 0 & 0\end{array}$

(1)

(3) 


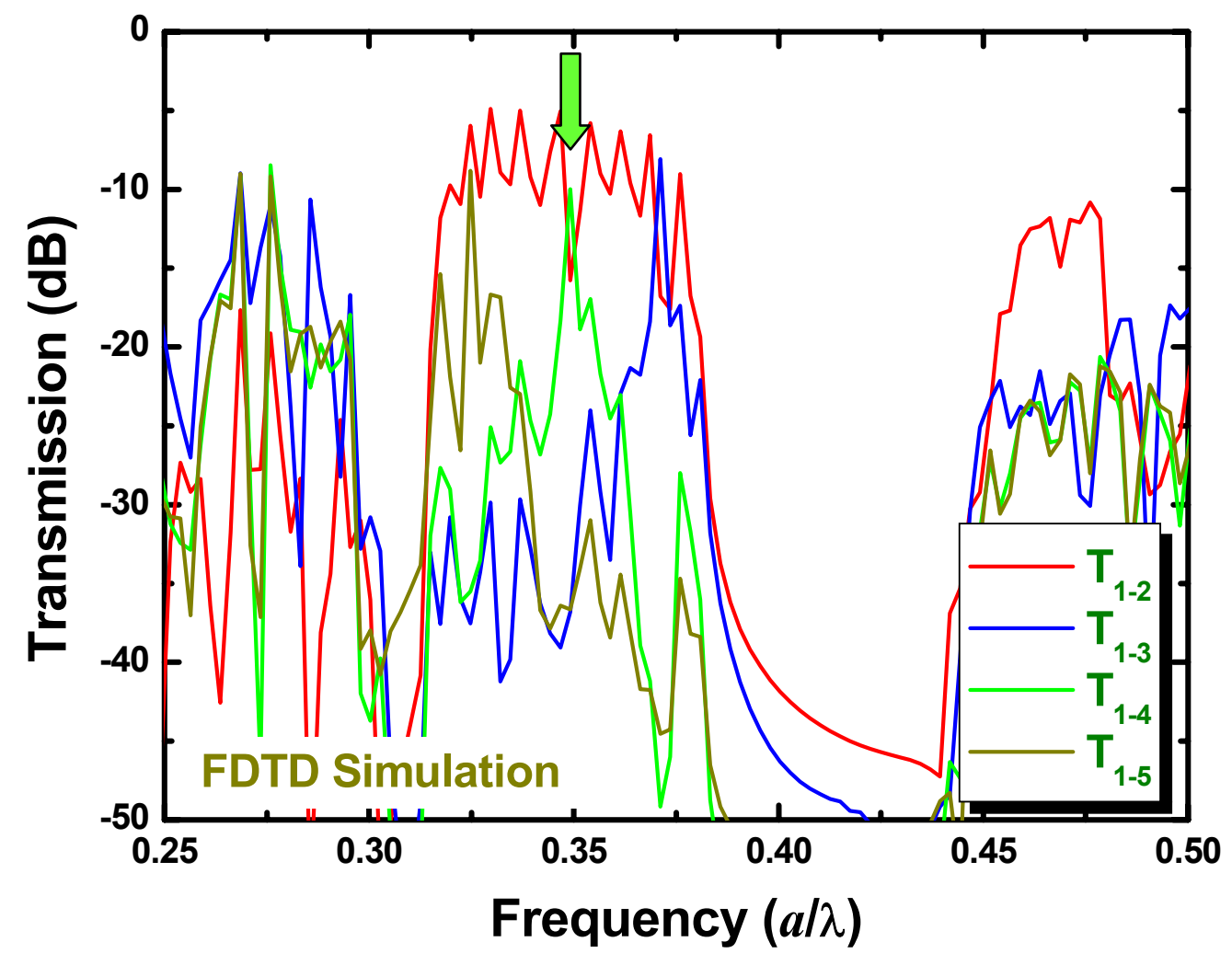

(2) 个

(5)

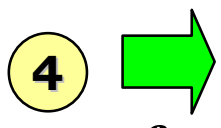

$\begin{array}{lllllllllll}0 & 0 & 0 & 0 & 0 & 0 & 0 & 0 & 0 & & f_{k} \\ 0 & 0 & 0 & & 0 & 0 & 0 & 0 & & \mathbf{f}_{k}\end{array}$

$\begin{array}{lllllllll}0 & 0 & 0 & 0 & 0 & 0 & 0 & 0 & 0 \\ 0 & 0 & 0 & & & 0 & 0 & 0 & 0 \\ 0 & 0 & 0 & 0 & 0 & 0 & 0 & 0 & 0 \\ 0 & 0 & 0 & & & 0 & & 0 & 0 \\ 0 & 0 & 0 & 0 & 0 & 0 & 0 & 0 & 0 \\ 0 & 0 & 0 & & & 0 & 0 & 0 & 0 \\ 0 & 0 & 0 & 0 & 0 & 0 & 0 & 0 & 0 \\ 0 & 0 & 0 & & & 0 & 0 & 0 & 0 \\ 0 & 0 & 0 & 0 & 0 & 0 & 0 & 0 & 0\end{array}$

(3)

(1) 

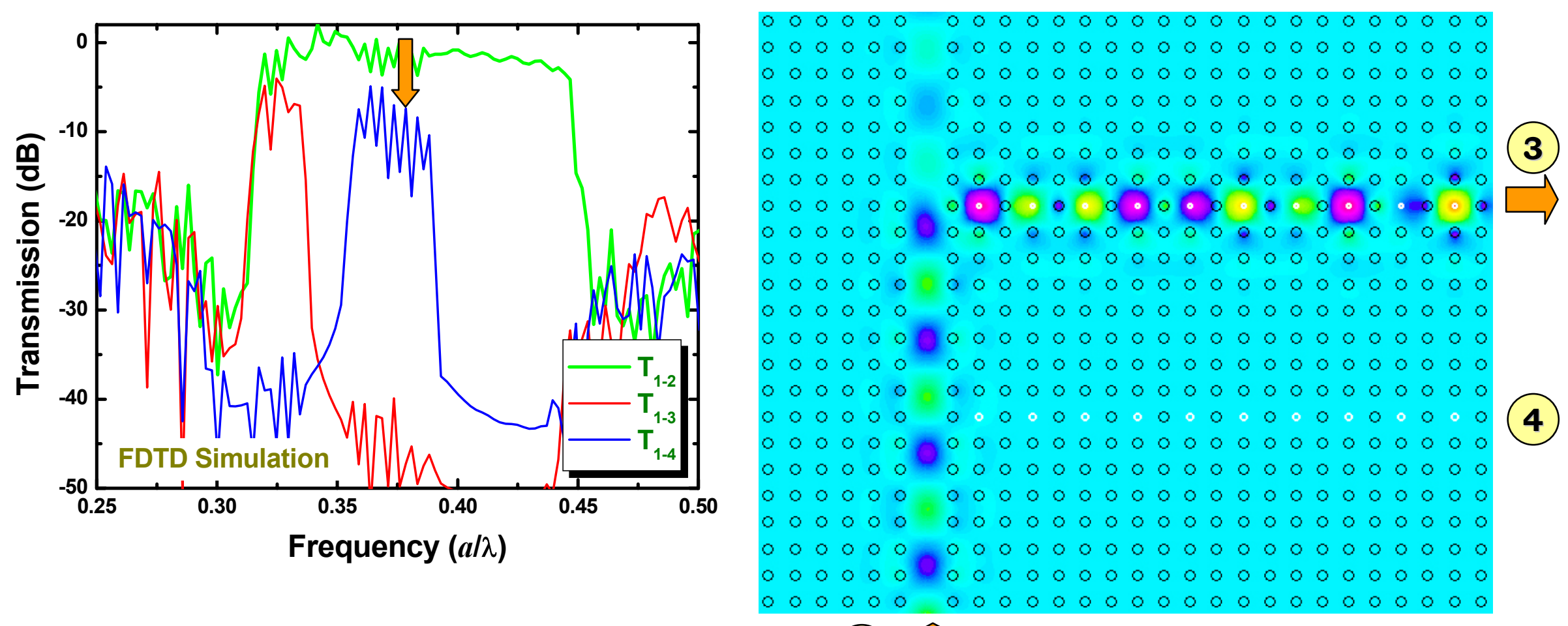

(1) $\widehat{~}$ 


\section{(2)}
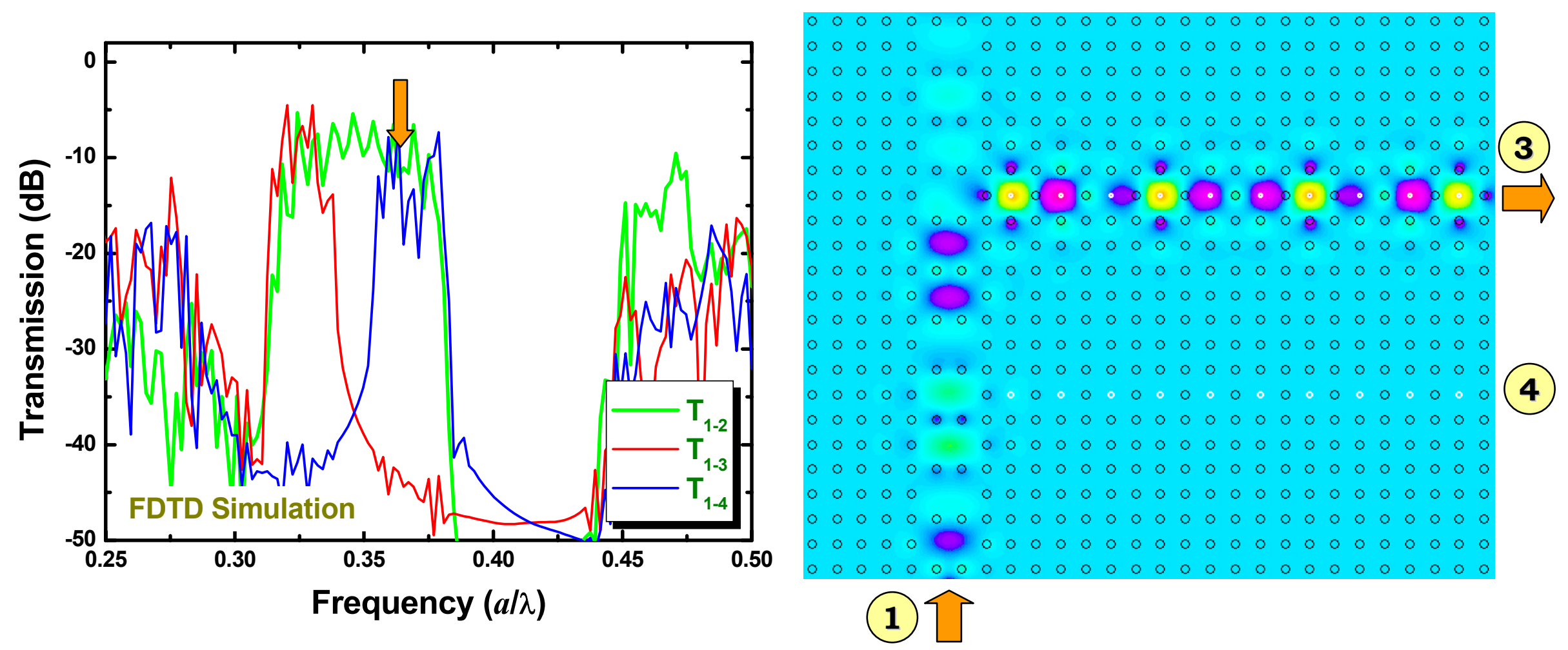
COUPLED WAVEGUIDES: DIRECTIONAL COUPLERS

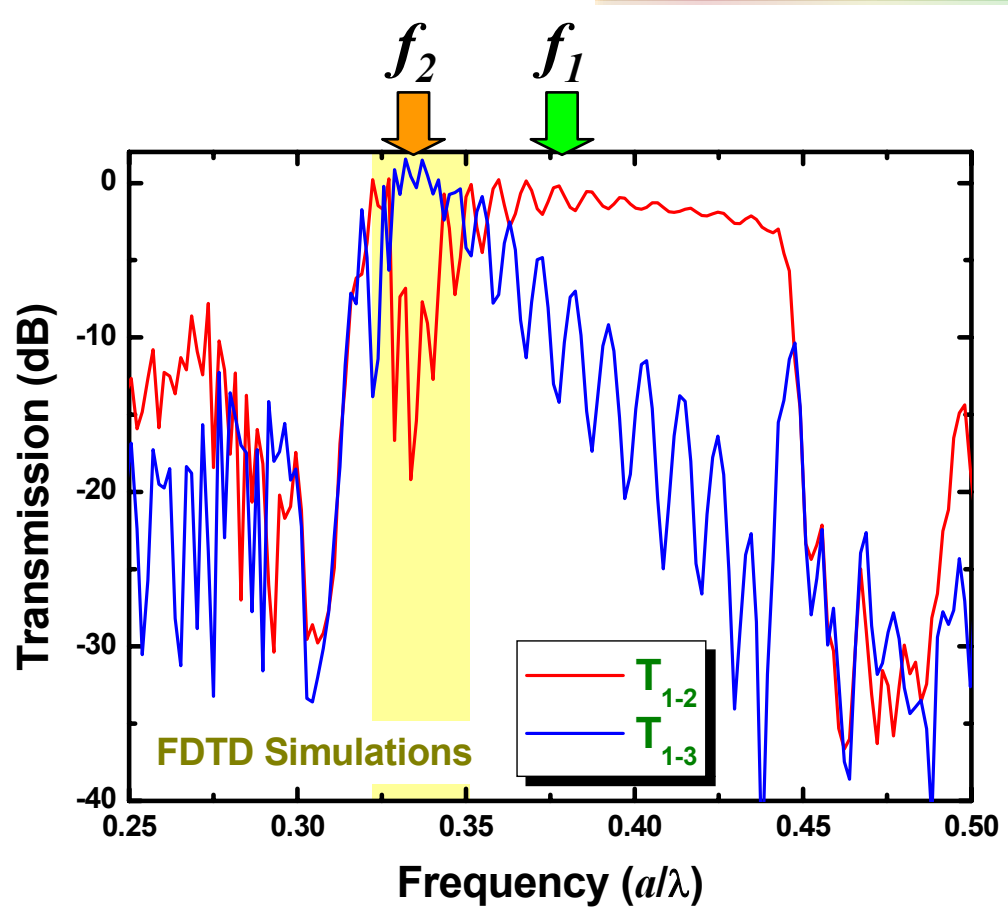

(2)

(3)

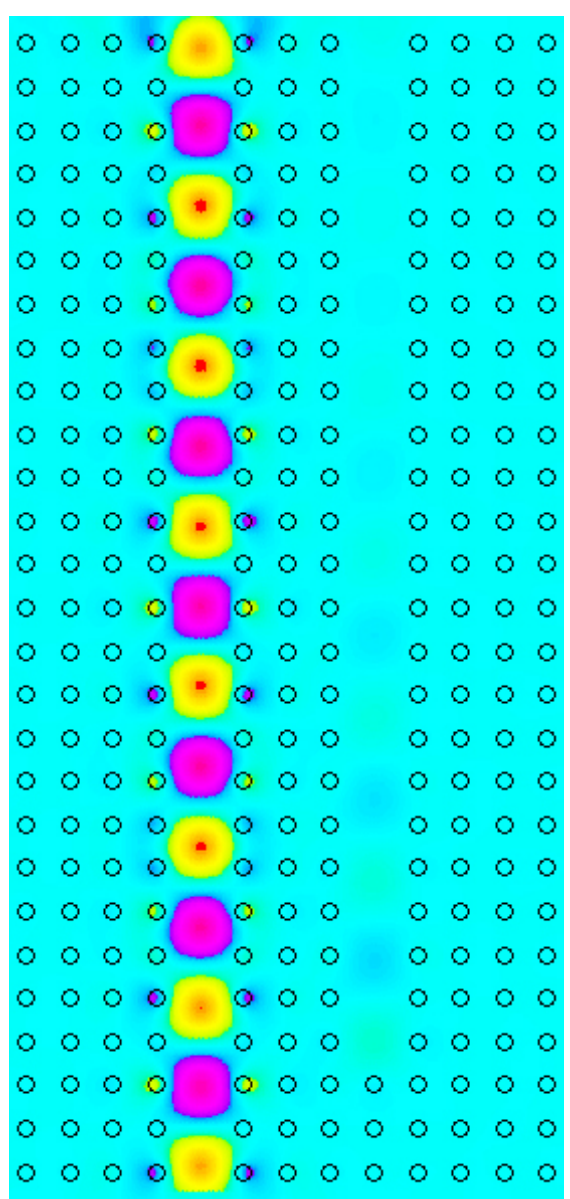

(1) $\widehat{U} f_{1}$
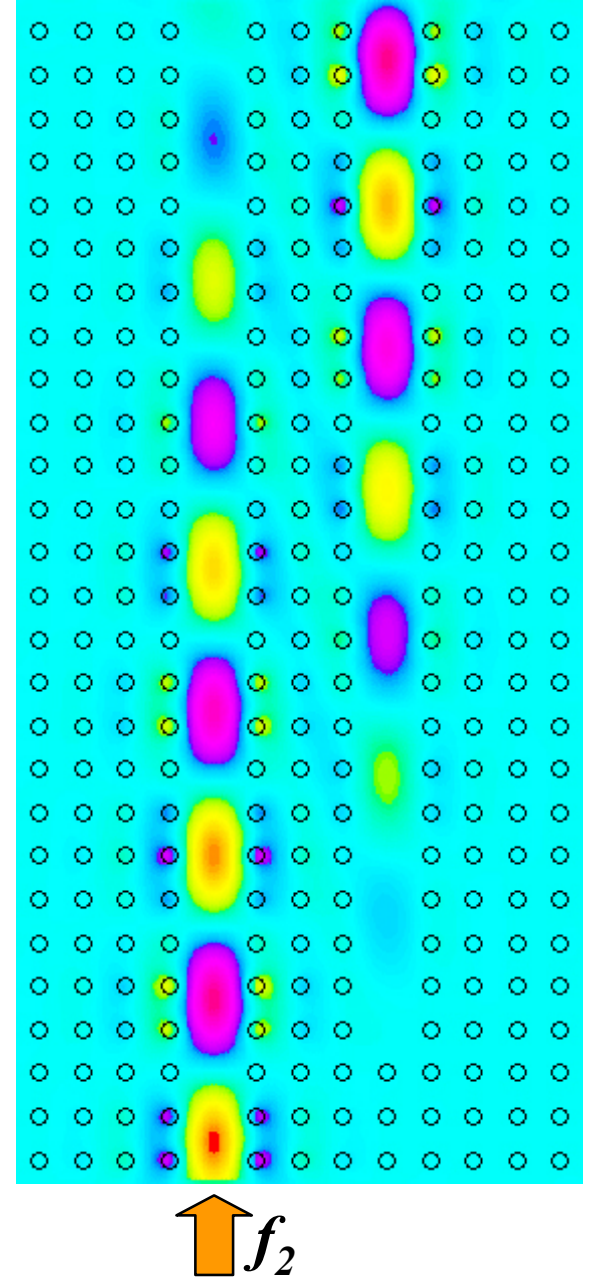

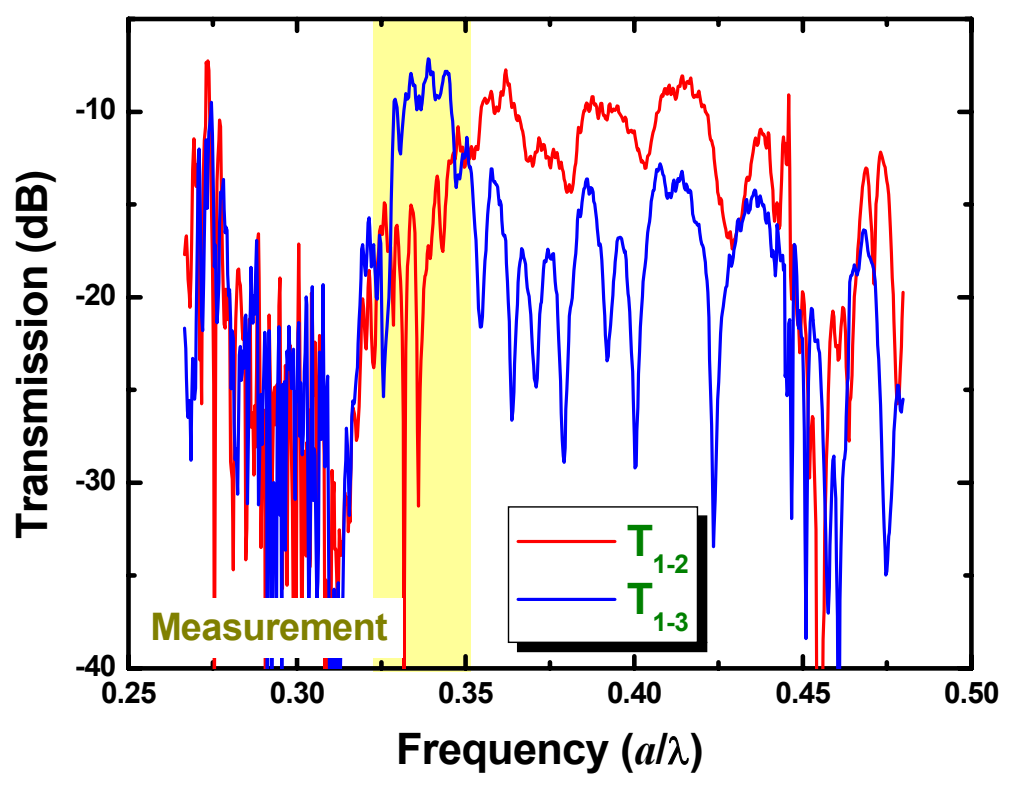

Bayindir and Ozbay, Optics Express [in preparation] 


\section{DIRECTIONAL COUPLERS: COUPLED-CAVITY WAVEGUIDES}

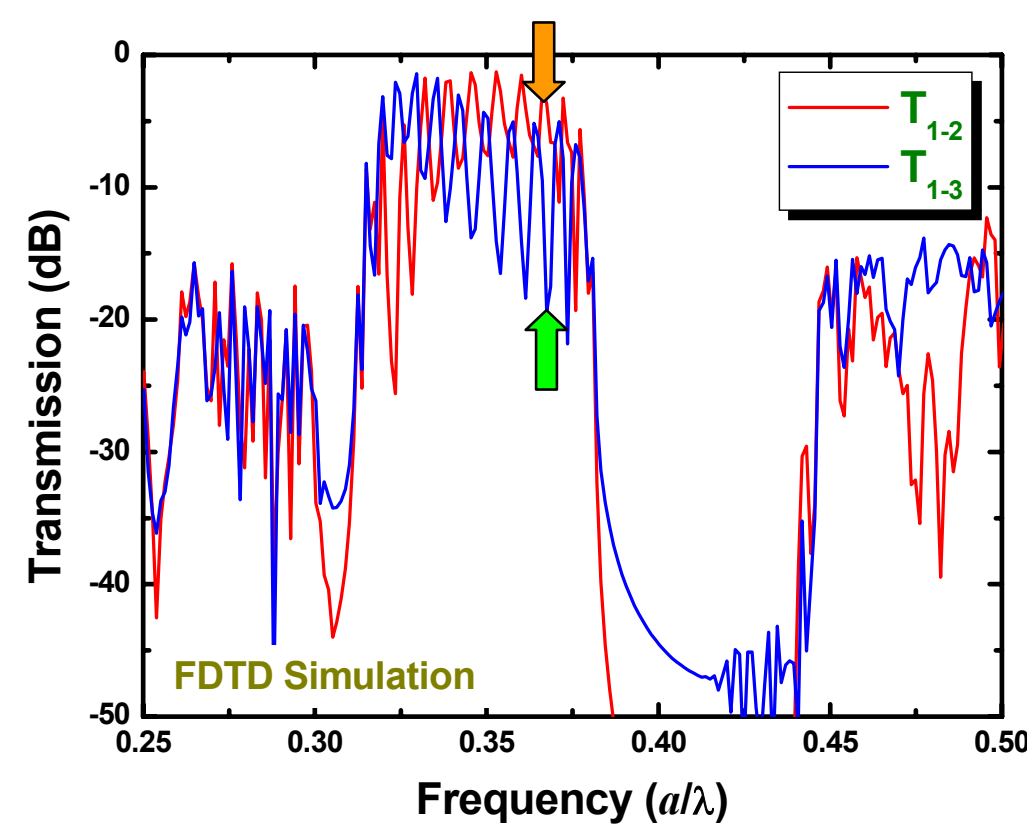

(2)

(3)
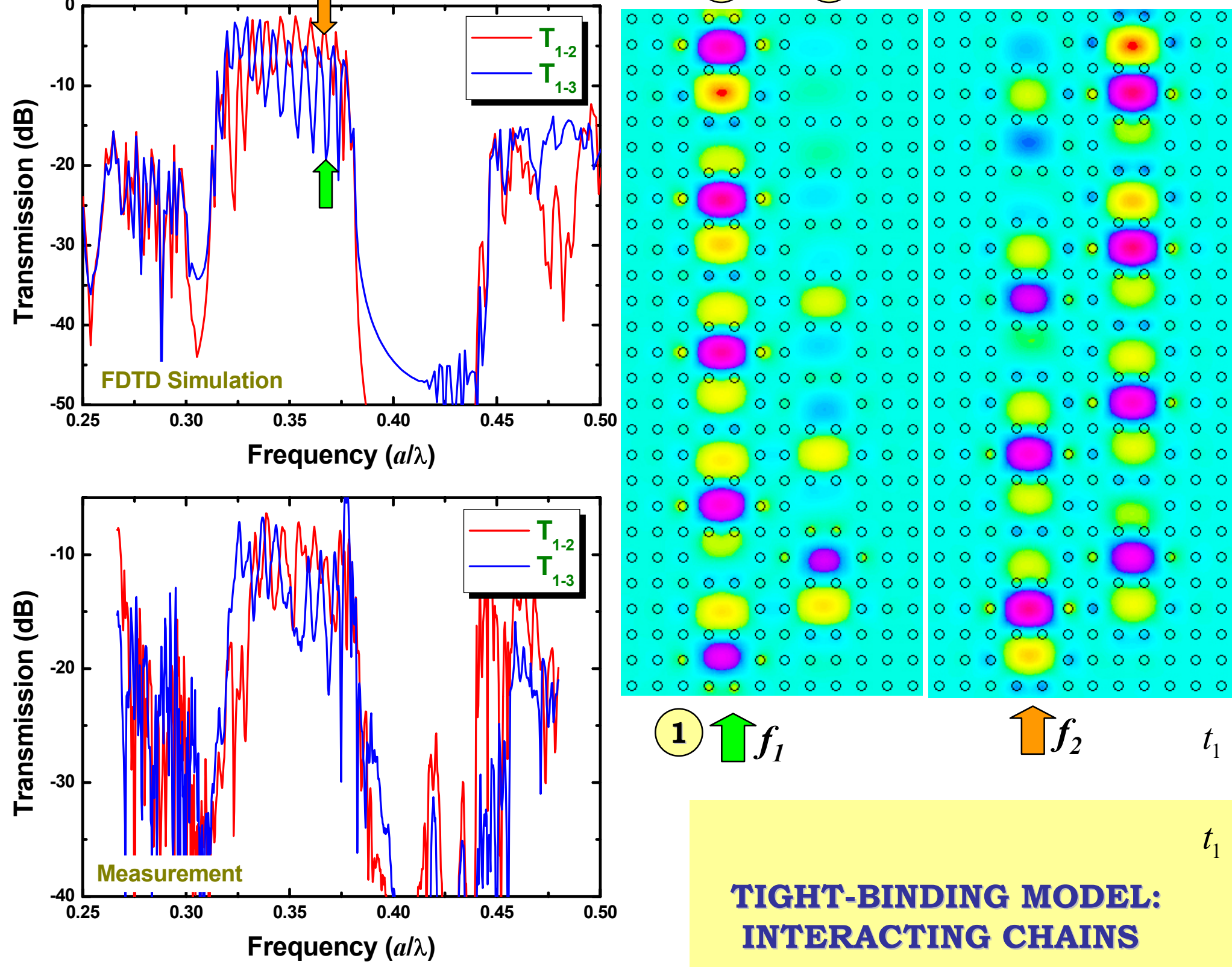

(1) $f_{1}$

$\widehat{\bigcup f_{2}}$

TIGHT-BINDING MODEL: INTERACTING CHAINS

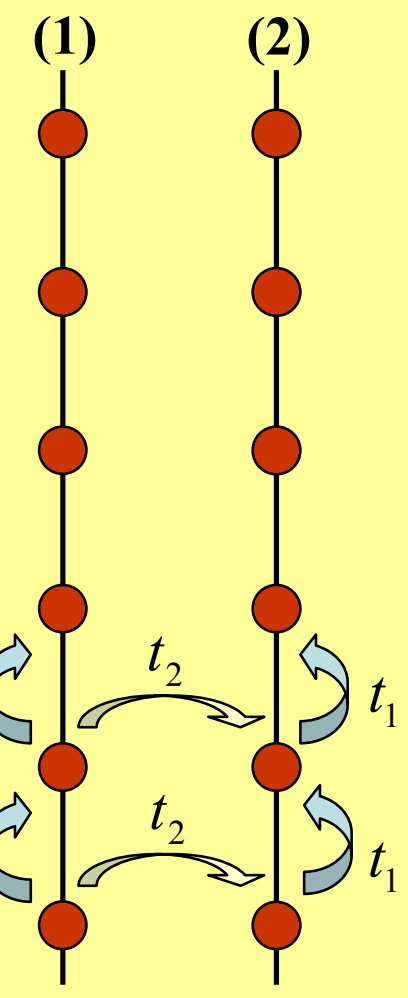

$$
t_{2}<<t_{1}
$$




\section{INCREASING EFFICIENCY OF NONLINEAR PROCESESS}
$\eta \propto 1 / v_{g}$
$v_{g} \rightarrow 0$
Large gain

\section{DISPERSION COMPENSATORS}

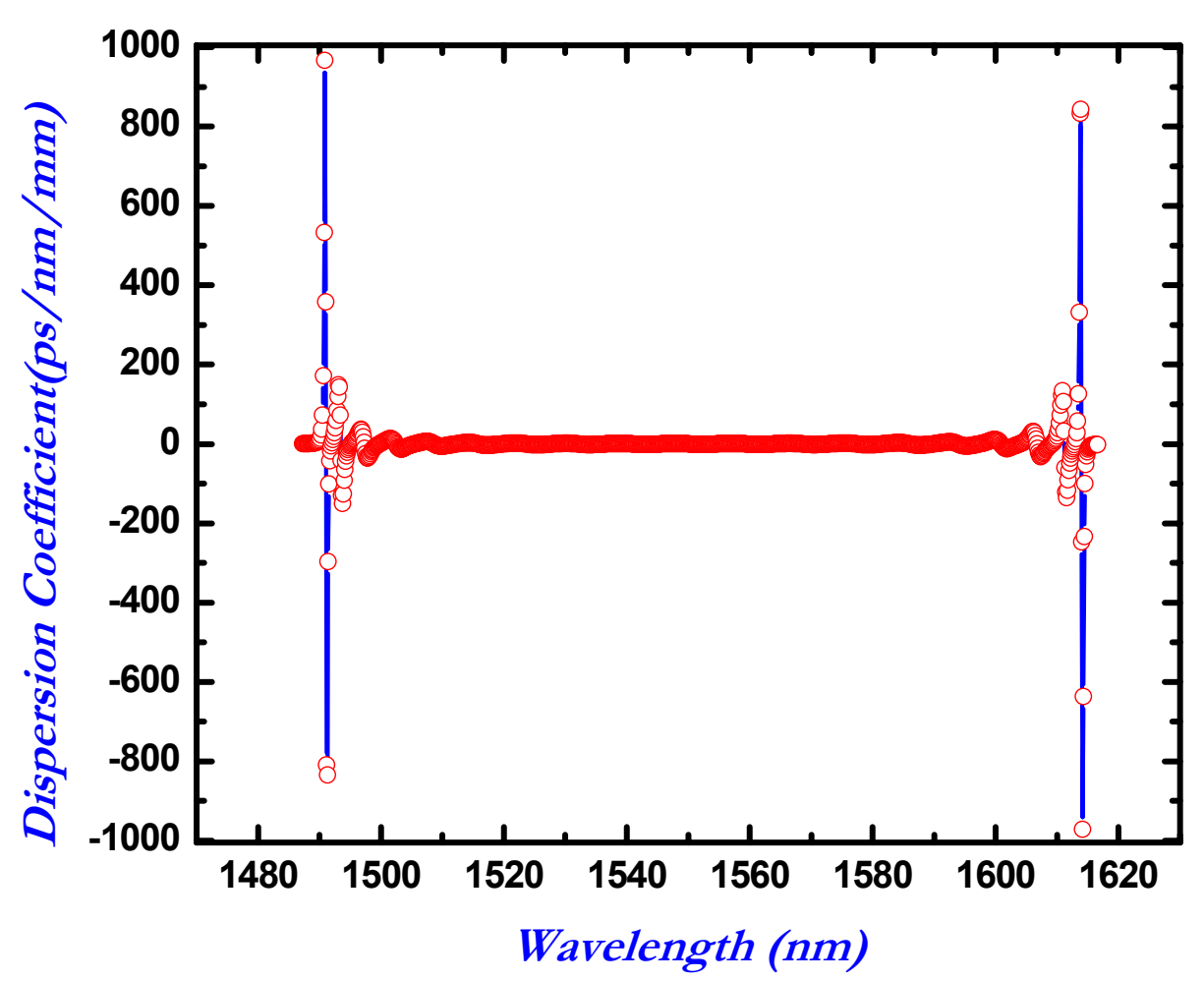

$$
D=-\frac{2 \pi c}{\lambda^{2}} \frac{d^{2} k}{d \omega^{2}}
$$




\section{QUASIPERIODIC [ PENROSE ] PHOTONIC CRYSTALS}
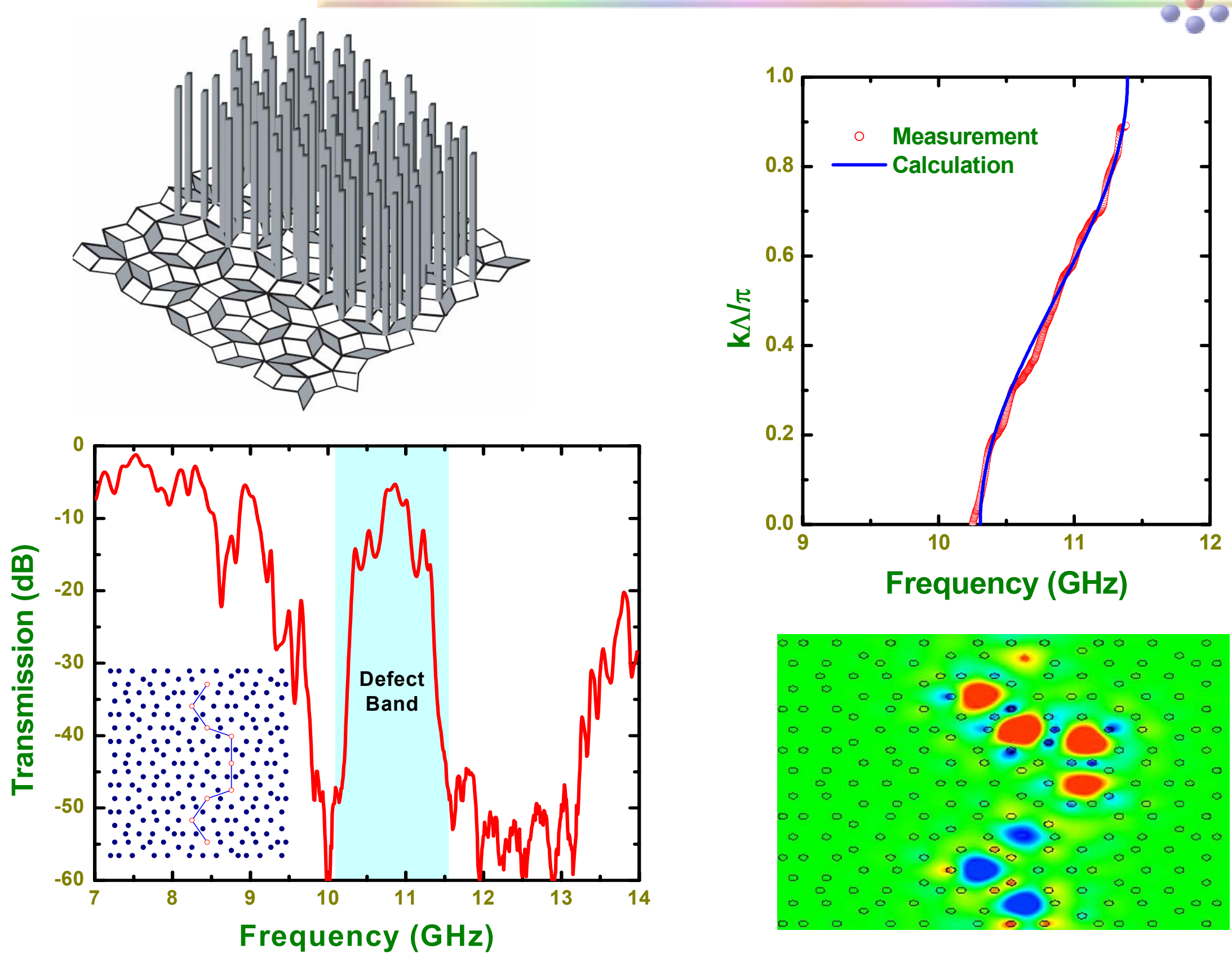
- periodic photonic crystal

O disordered photonic crystal

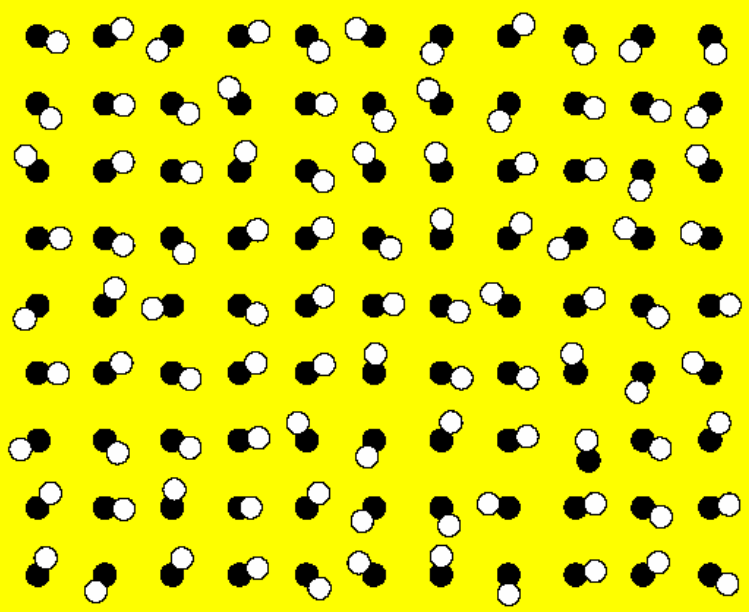

$$
z_{i} \rightarrow z_{i} r e^{i \varphi}
$$

$r$ : randomness parameter

$\varphi$ : random variable between $[0, \pi]$
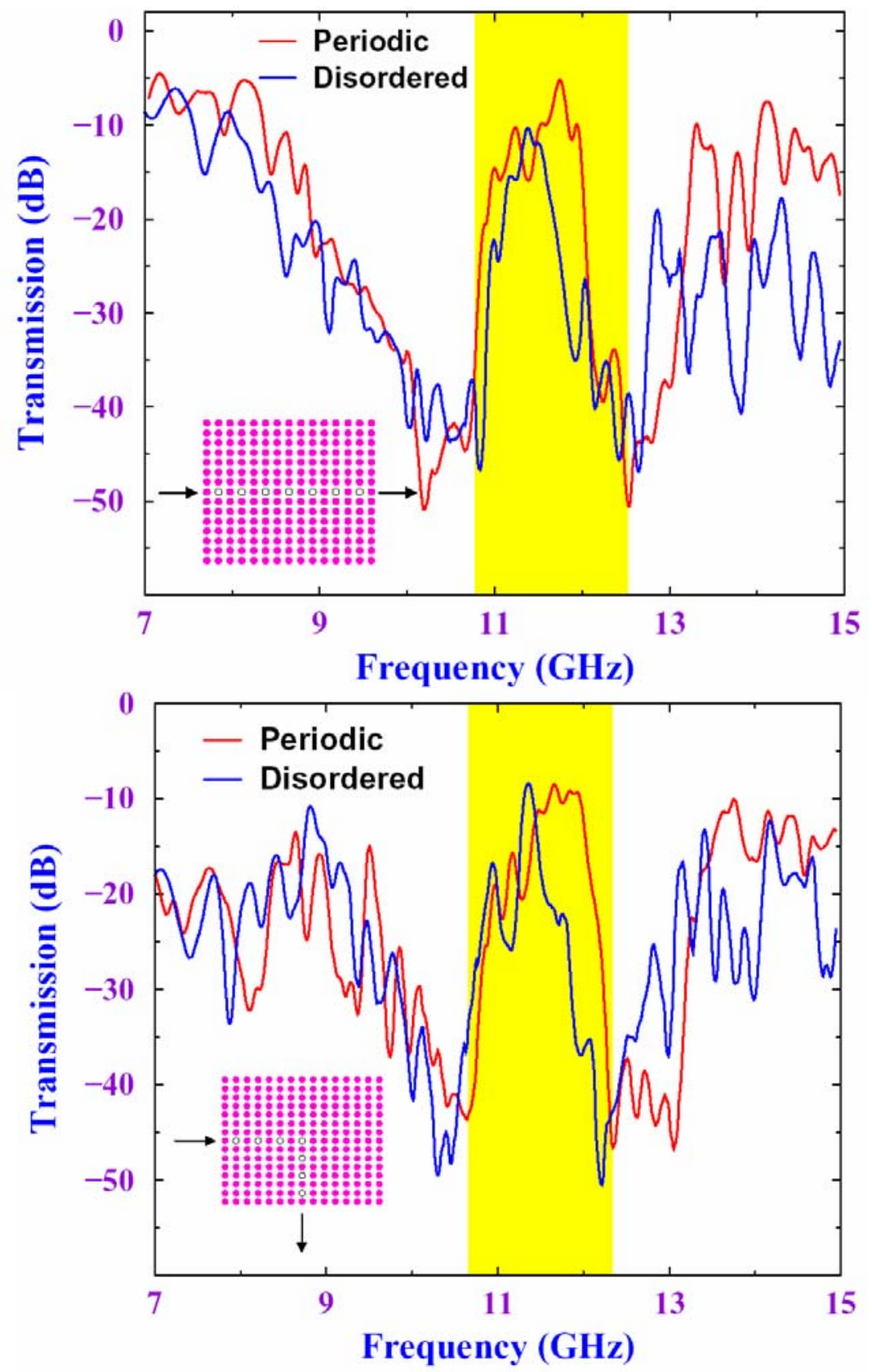


\section{D LAYER-BY-LAYER PHOTONIC CRYSTALS}

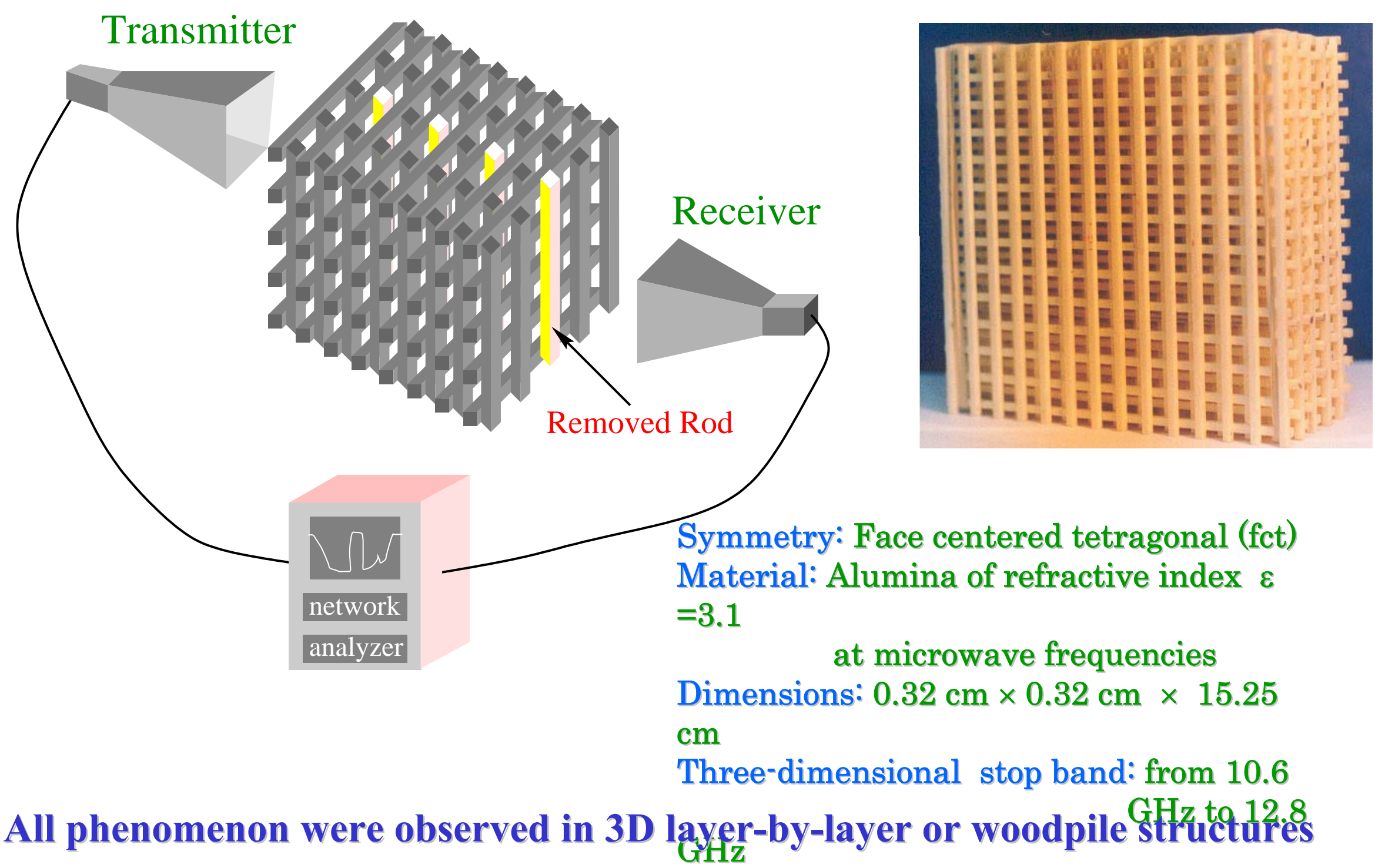

Bayindir, et al., Phys. Rev. Lett. 82, 2140 (2000); Bayindir, et al., Phys. Rev. B 61, R11855 (2000) 
COUPLED-CAVITIES IN 1D STRUCTURES

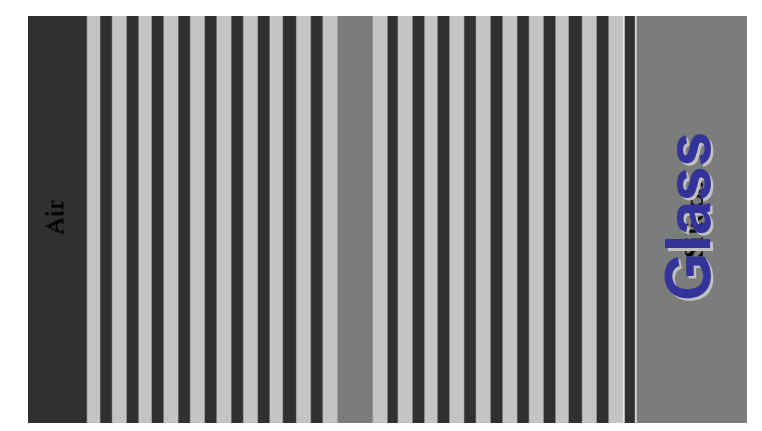

$\begin{array}{lcc} & n & d[ \\ \mathrm{Si}_{3} \mathrm{~N}_{4} & \mathbf{2 . 1 0} & \mathbf{7 0} \\ \mathrm{SiO}_{2} & \mathbf{1 . 4 7} & \mathbf{9 7}\end{array}$

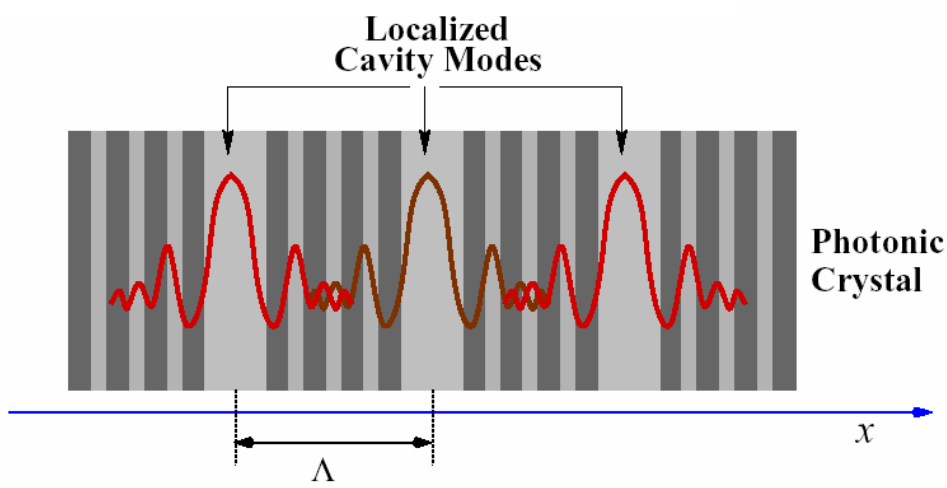

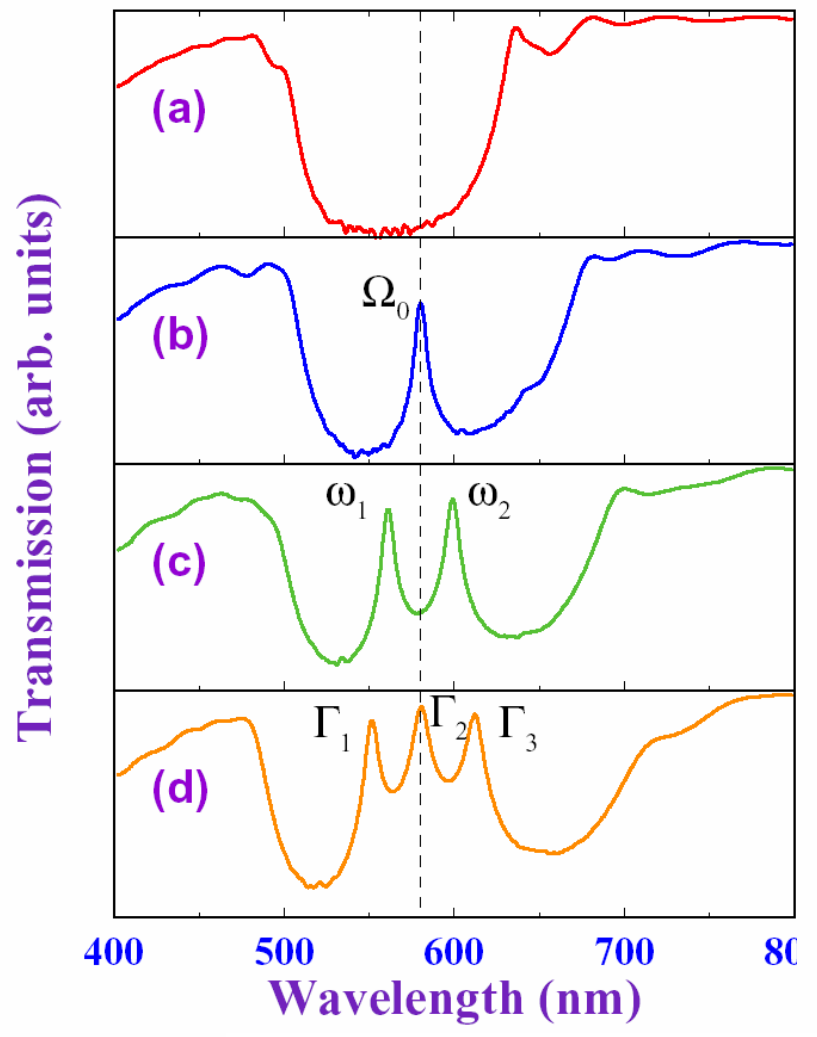

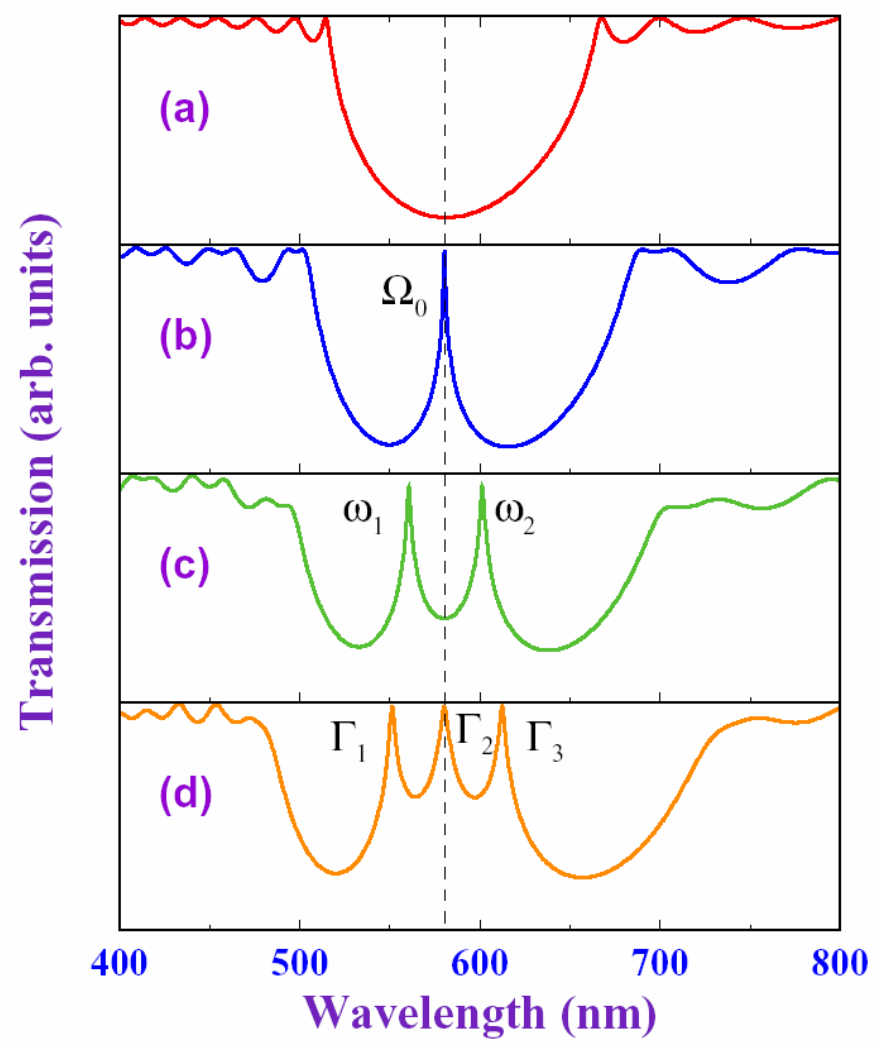

Bayindir, et al., Appl. Phys. A 72, 117 (2001); Appl. Phys. A 73, 125 (2001)

Experimental results agree well with TMM and TB predictions for the three coupled-cavities 


\section{COUPLED-CAVITIES IN 1D STRUCTURES}
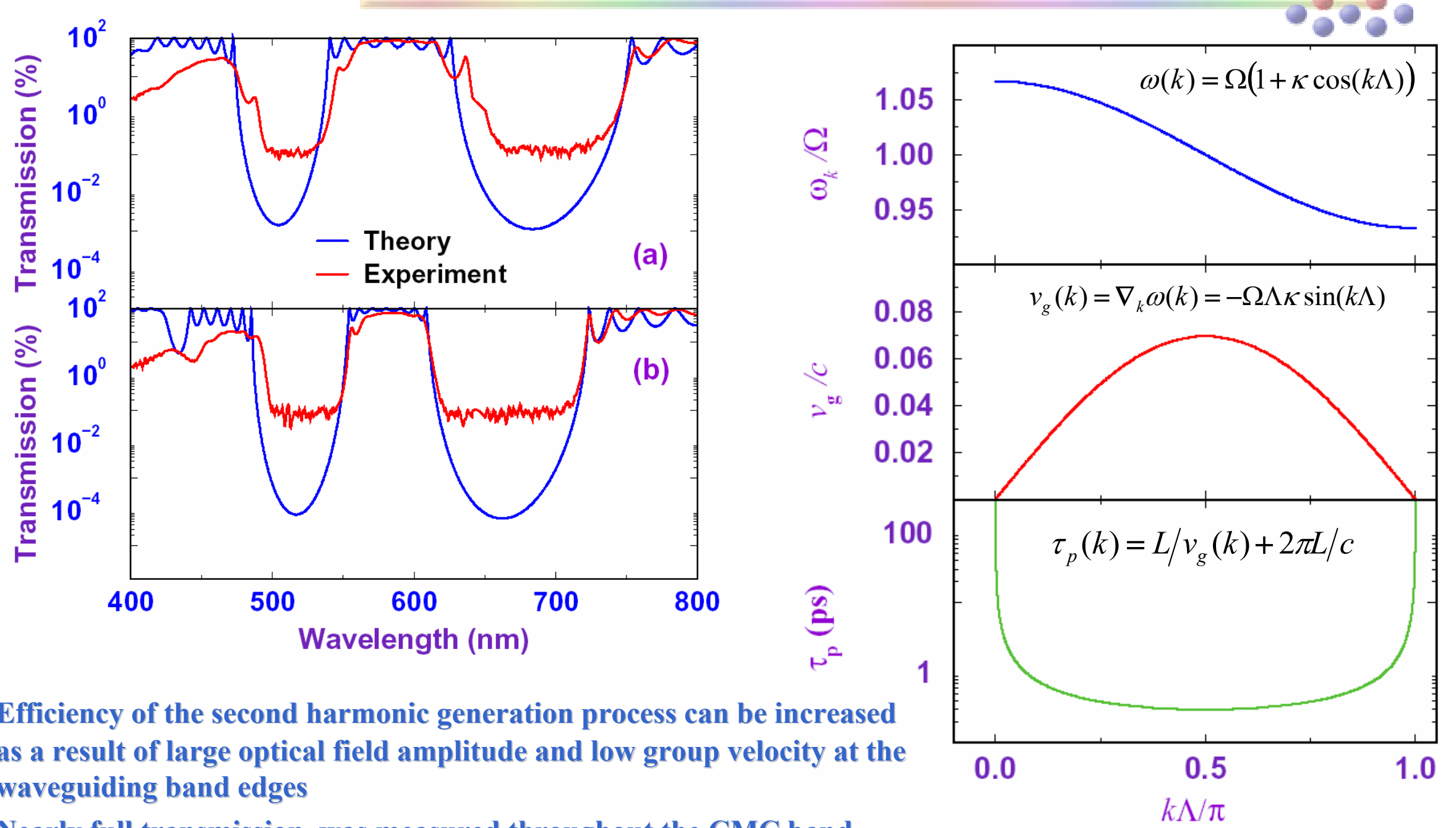

- Efficiency of the second harmonic generation process can be increased as a result of large optical field amplitude and low group velocity at the waveguiding band edges

- Nearly full transmission was measured throughout the CMC band

- The transfer matrix method results agree well with the experimental observations

- The position and bandwidth of waveguiding band can be adjusted by changing the thicknesses of the layers and the distance between the cavity layers

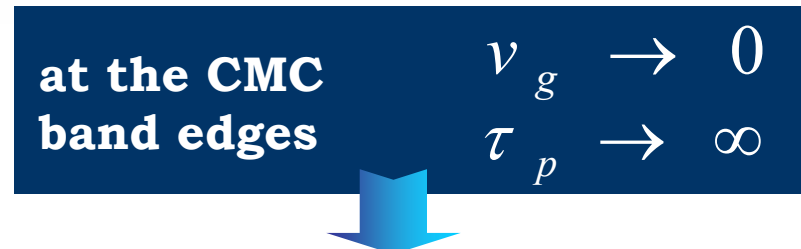




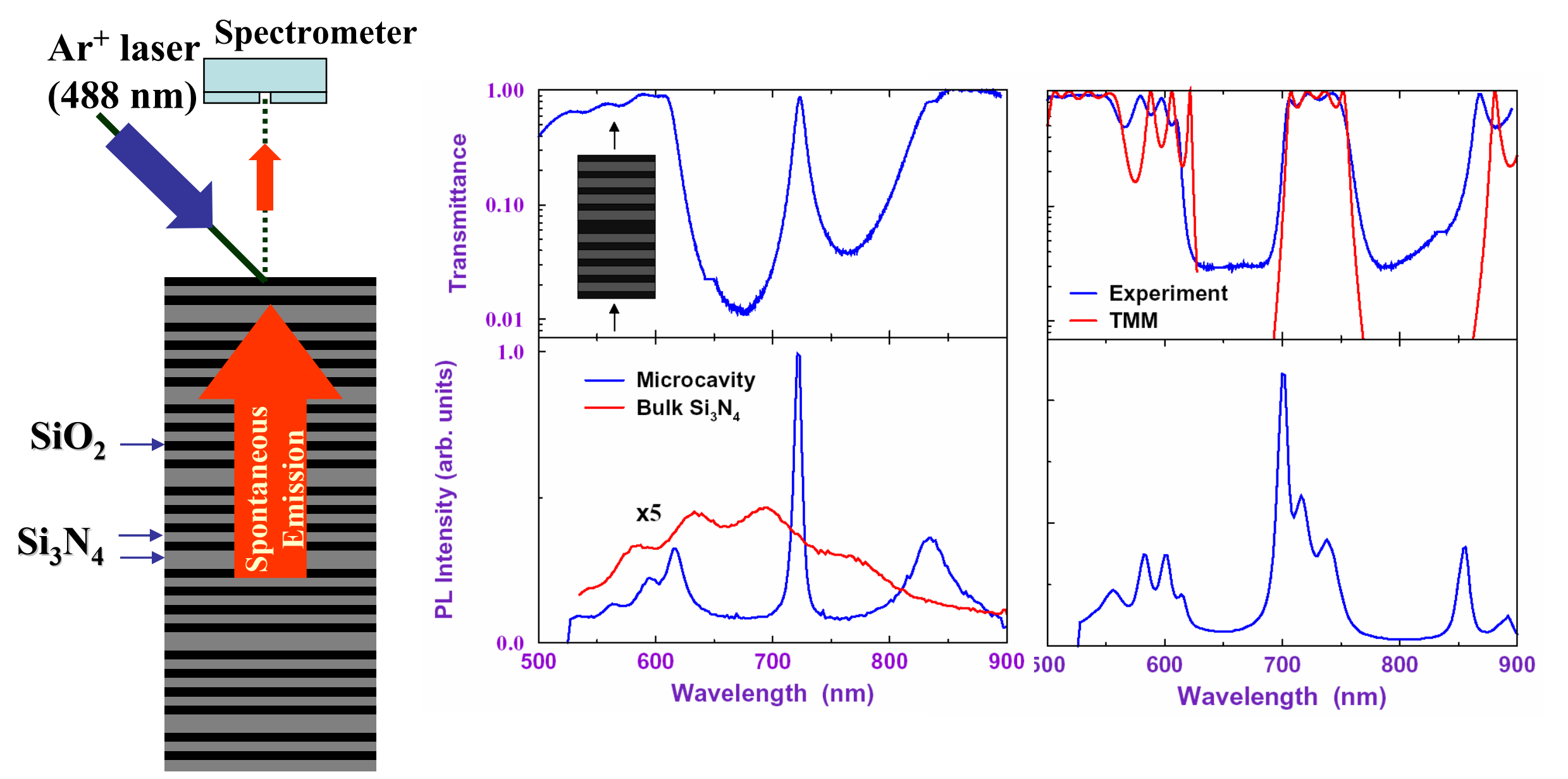

- The photoluminescence spectrum was strongly modified in the presence of Fabry-Perot microcavity

- A strong spontaneous emission was achieved for a wide range of wavelengths

- The spontaneous emission was significantly enhanced at the coupled-cavity band edges 
Straight Waveguide

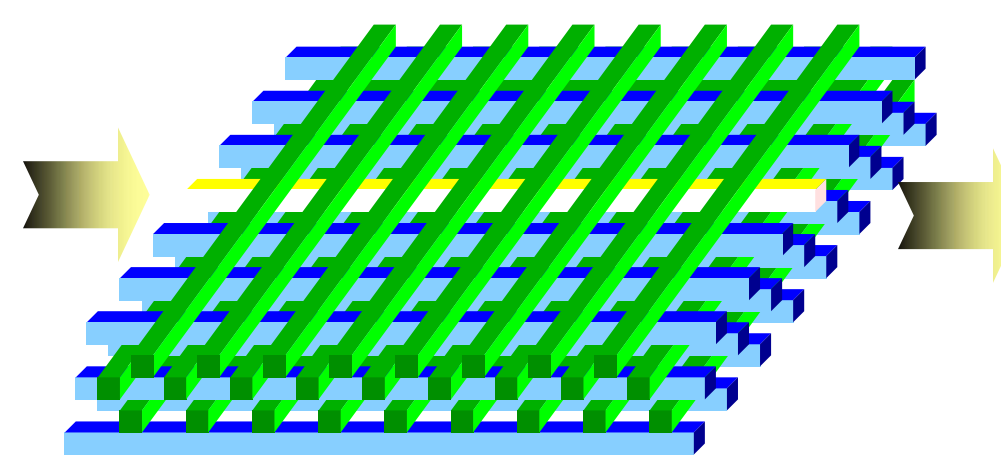

Power Splitter

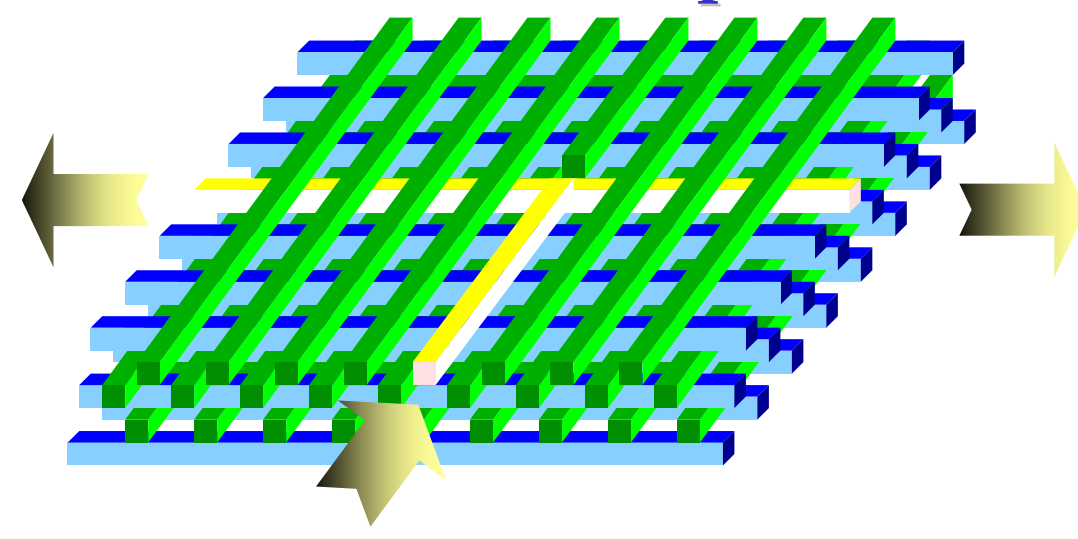

Bended Waveguide

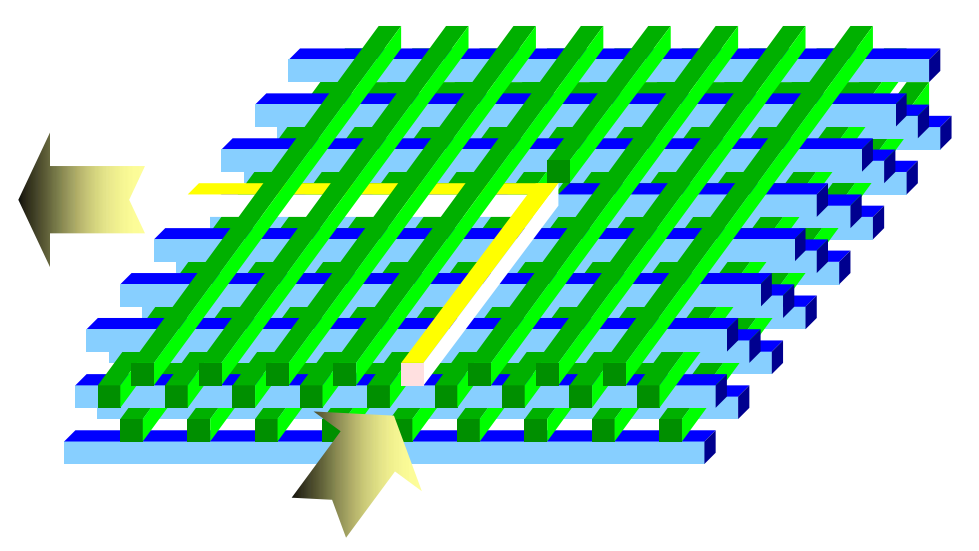

Noda et al, APL 75, 3739 1999;

Bayindir et al., PRB 63, 081107(R) (2001)

Full confinement of electromagnetic waves in 3D layer-by-layer photonic crystals 


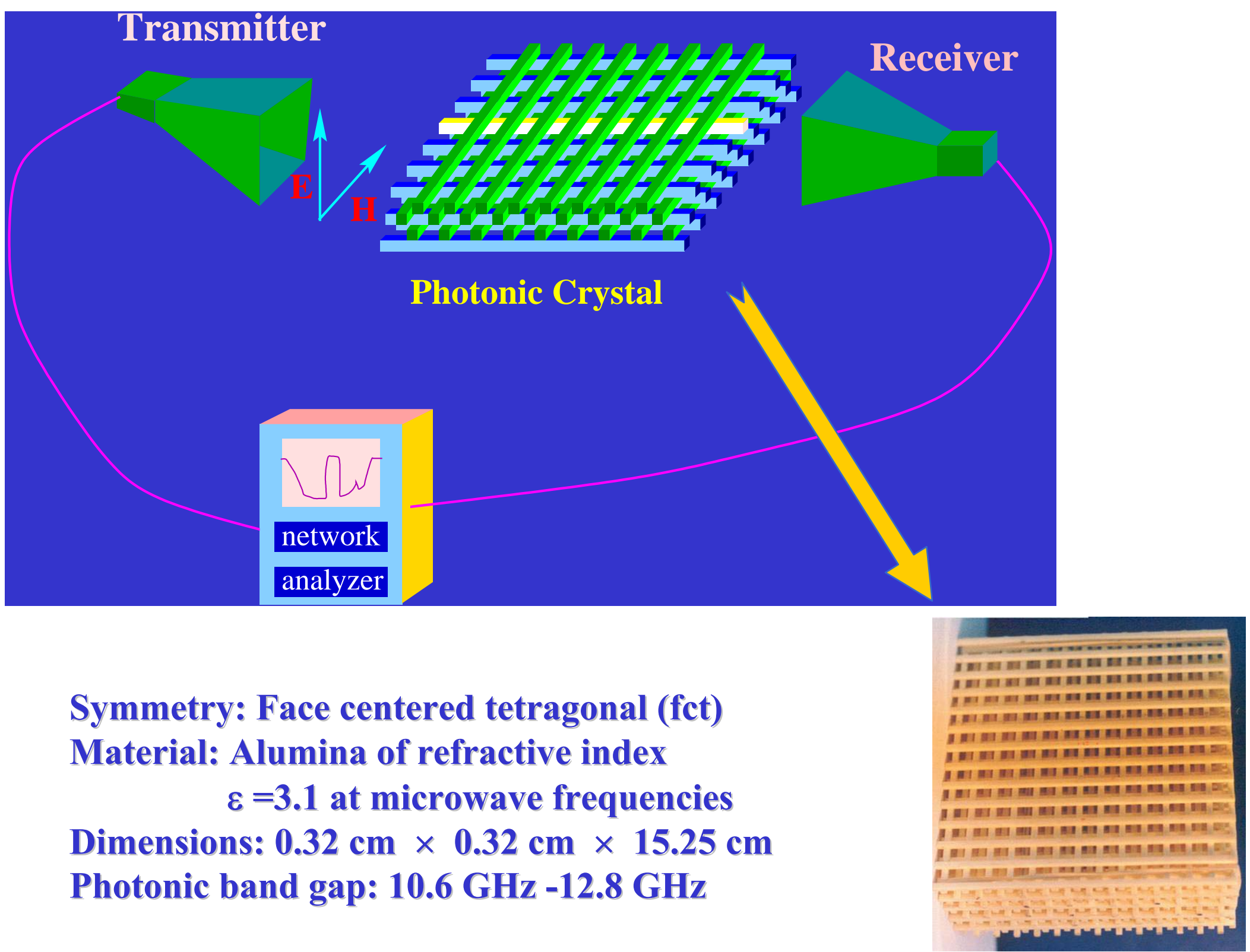



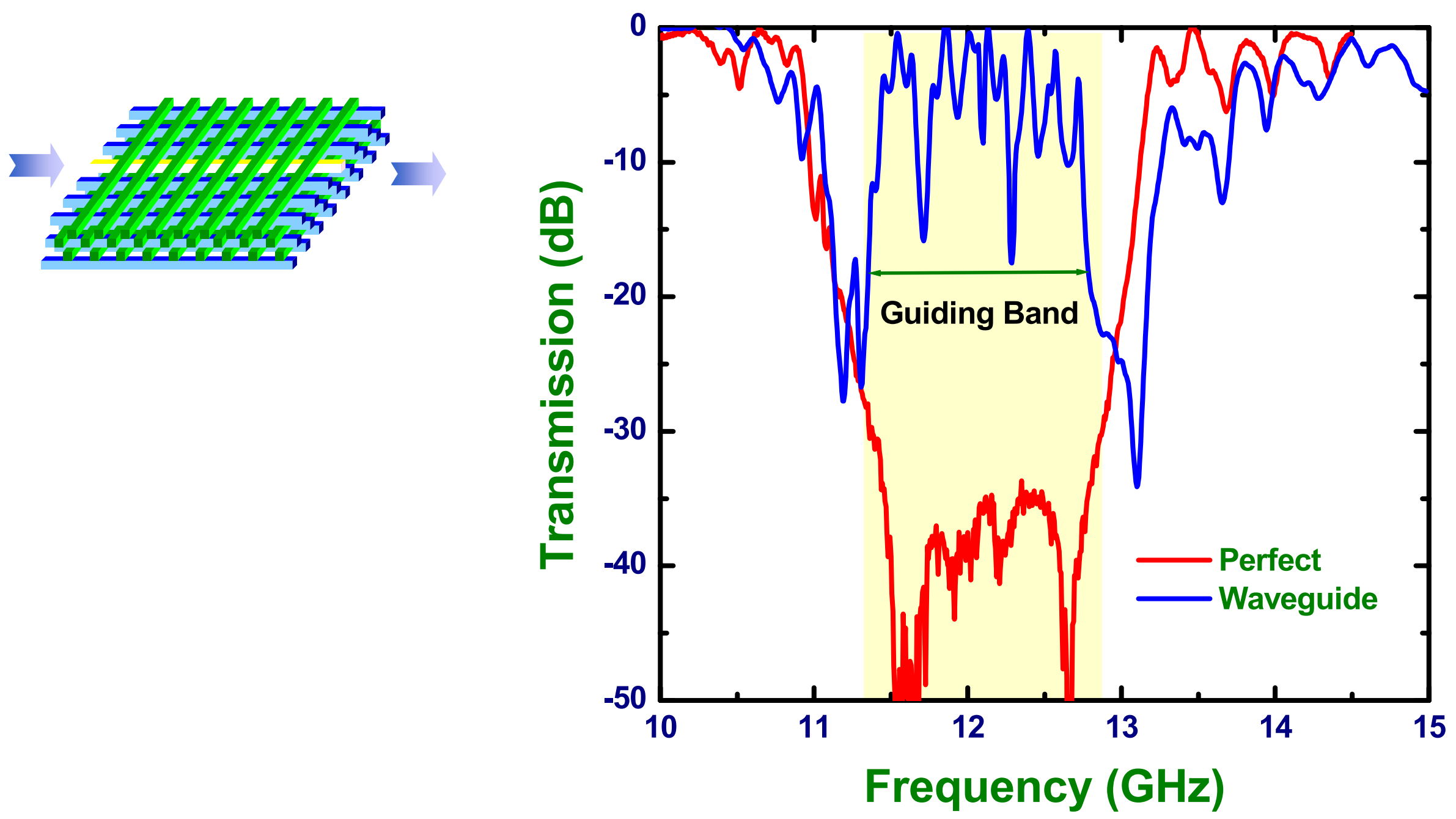

$>$ Nearly full transmission was achieved for certain firequencies

$>$ The full transmission within the waveguiding band was a proff of how well the wave was confined and guided without lossess 

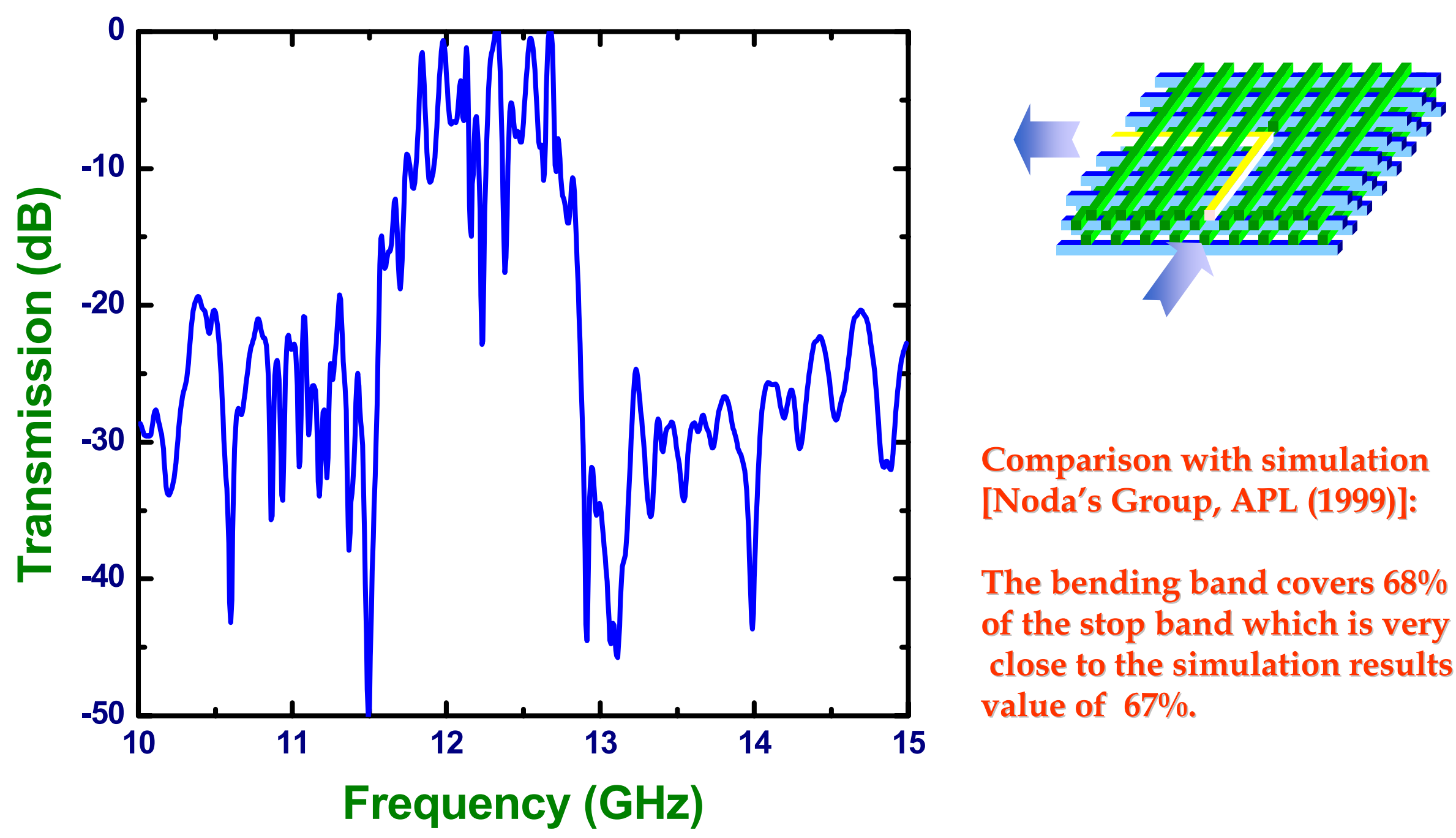

Comparison with simulation [Noda's Group, APL (1999)]:

The bending band covers $68 \%$ of the stop band which is very close to the simulation results value of $67 \%$.

15

$>$ The full transmission through a $90^{\circ}$ bent was achieved for certain frequencies throughout the waveguiding band 

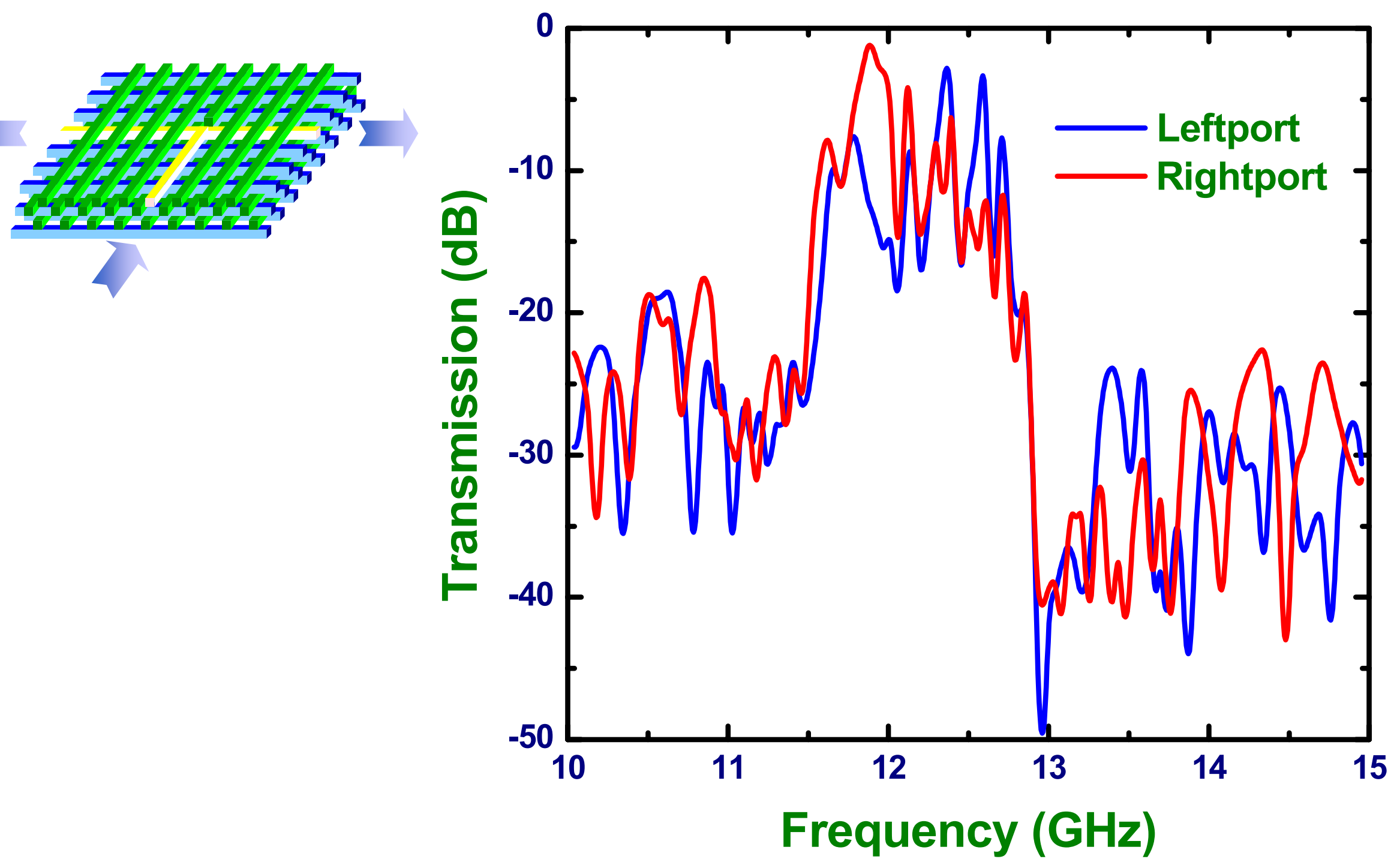

$>$ The electromagnetic power in the input port splits into the two output ports throughout the guiding band 


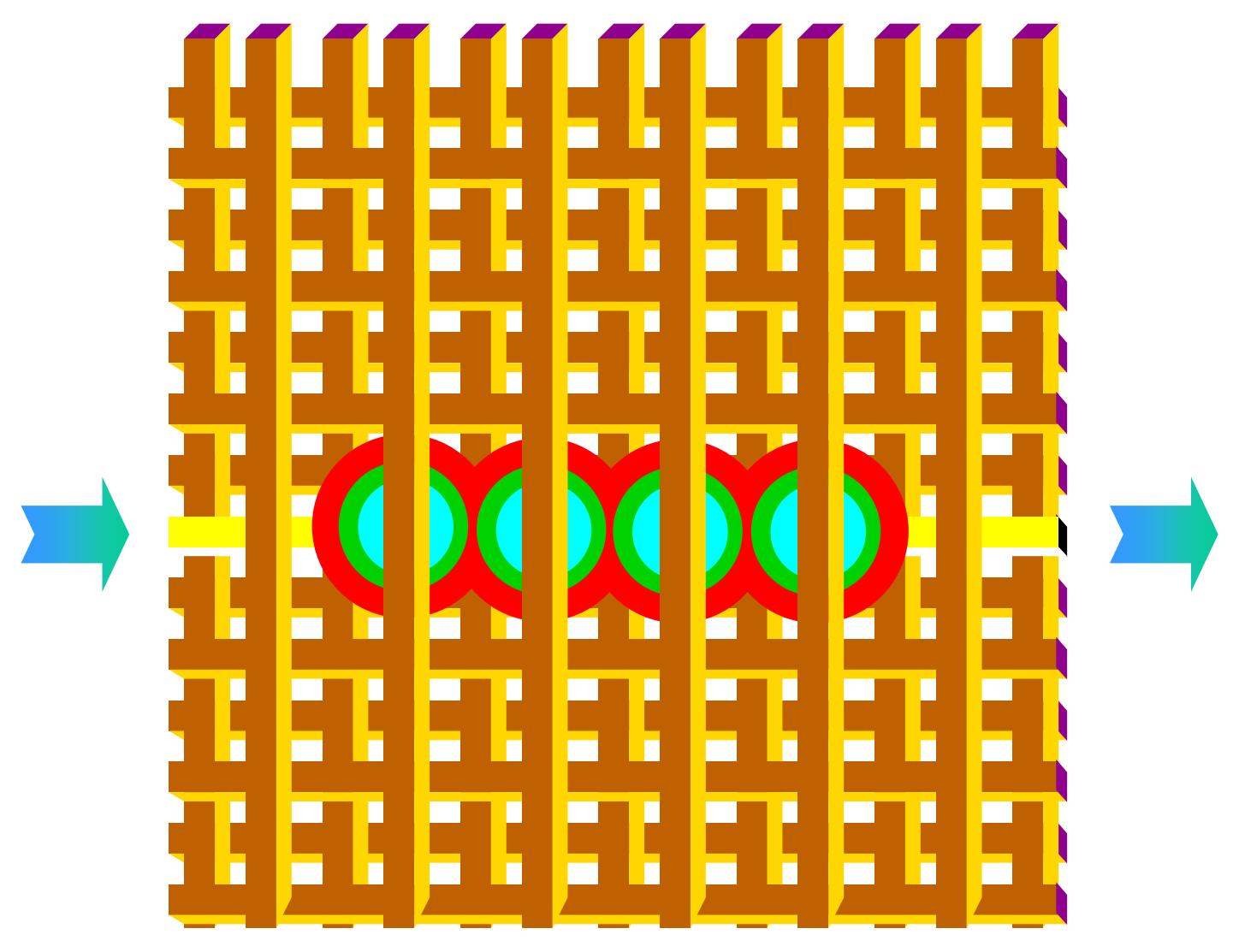

Each vacancy just below the removed rod behaves as a boxlike cavity

$>$ The coupling between these localized cavity modes allows propagation of photons by hopping through the vacancy of the missing rod 

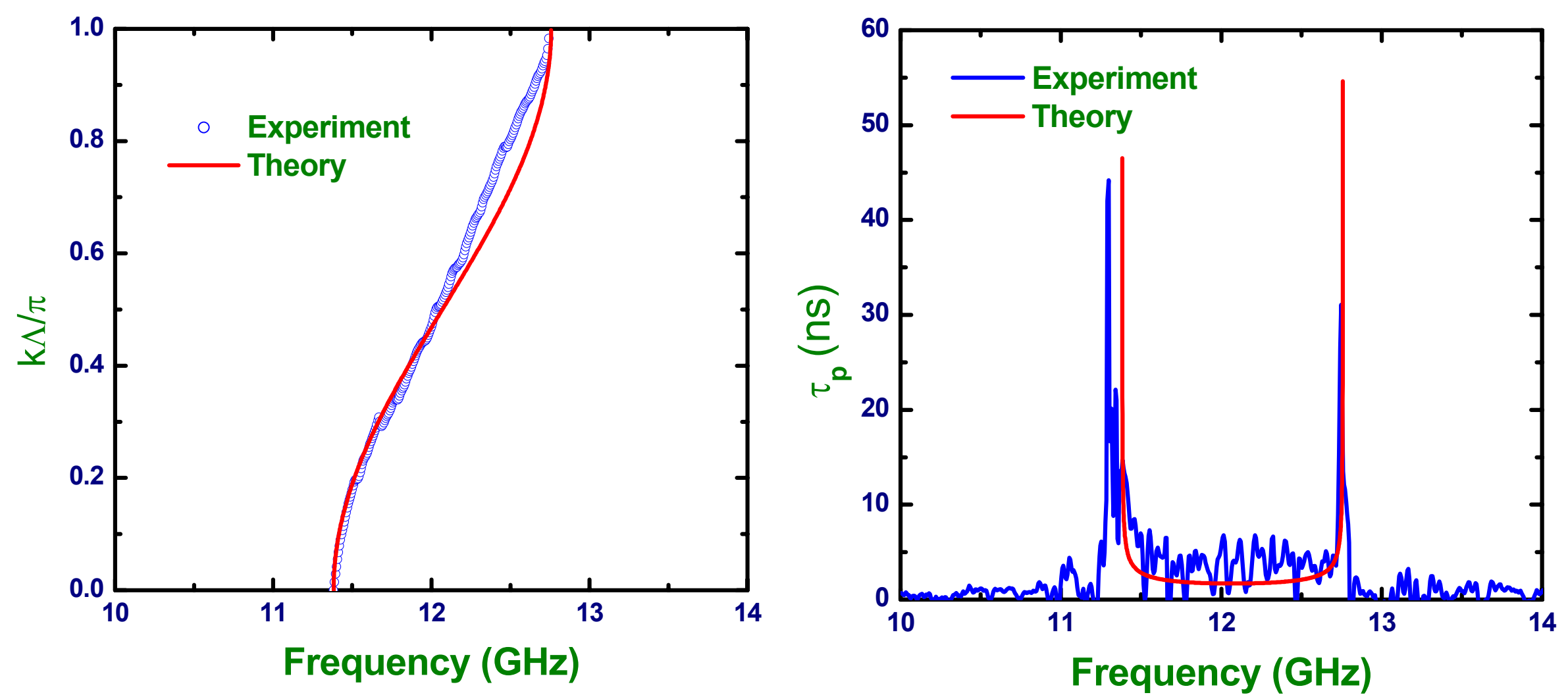

$$
\omega(k)=\Omega(1+\kappa \cos (k \Lambda))
$$

$$
\tau_{p}(k)=L / v_{g}(k)+2 \pi L / c
$$

Experimental results were in good agreement with the tight-binding approximation predictions 

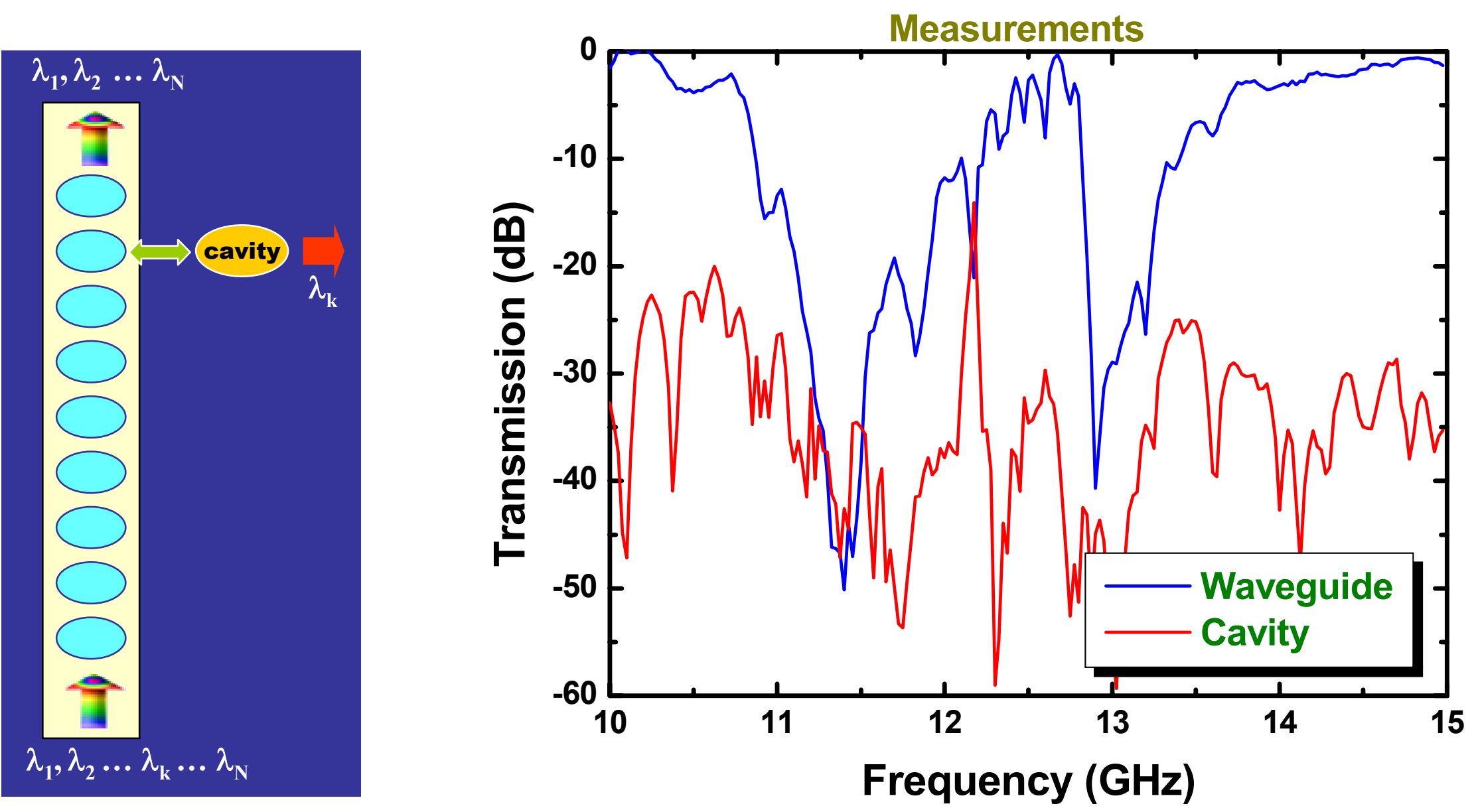

Bayindir and Ozbay [in preparation]

- Electromagnetic wave with a specifie frequency can dropped from the guided mode inside the waveguide.

४ Tunability can achieved by changing properties of the cavity. 
Lin et al, Nature 394, 251 (1998)

\section{5uH}

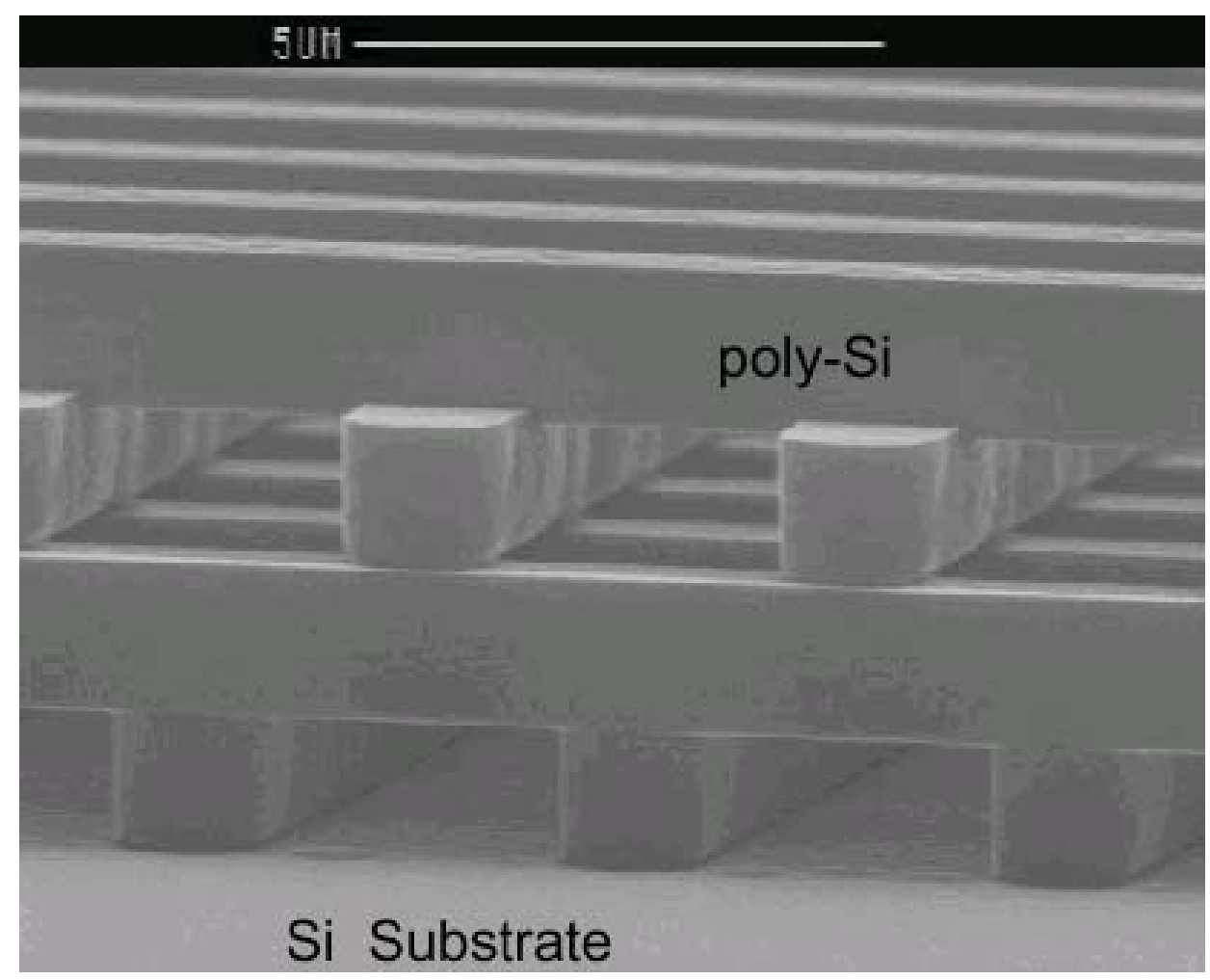

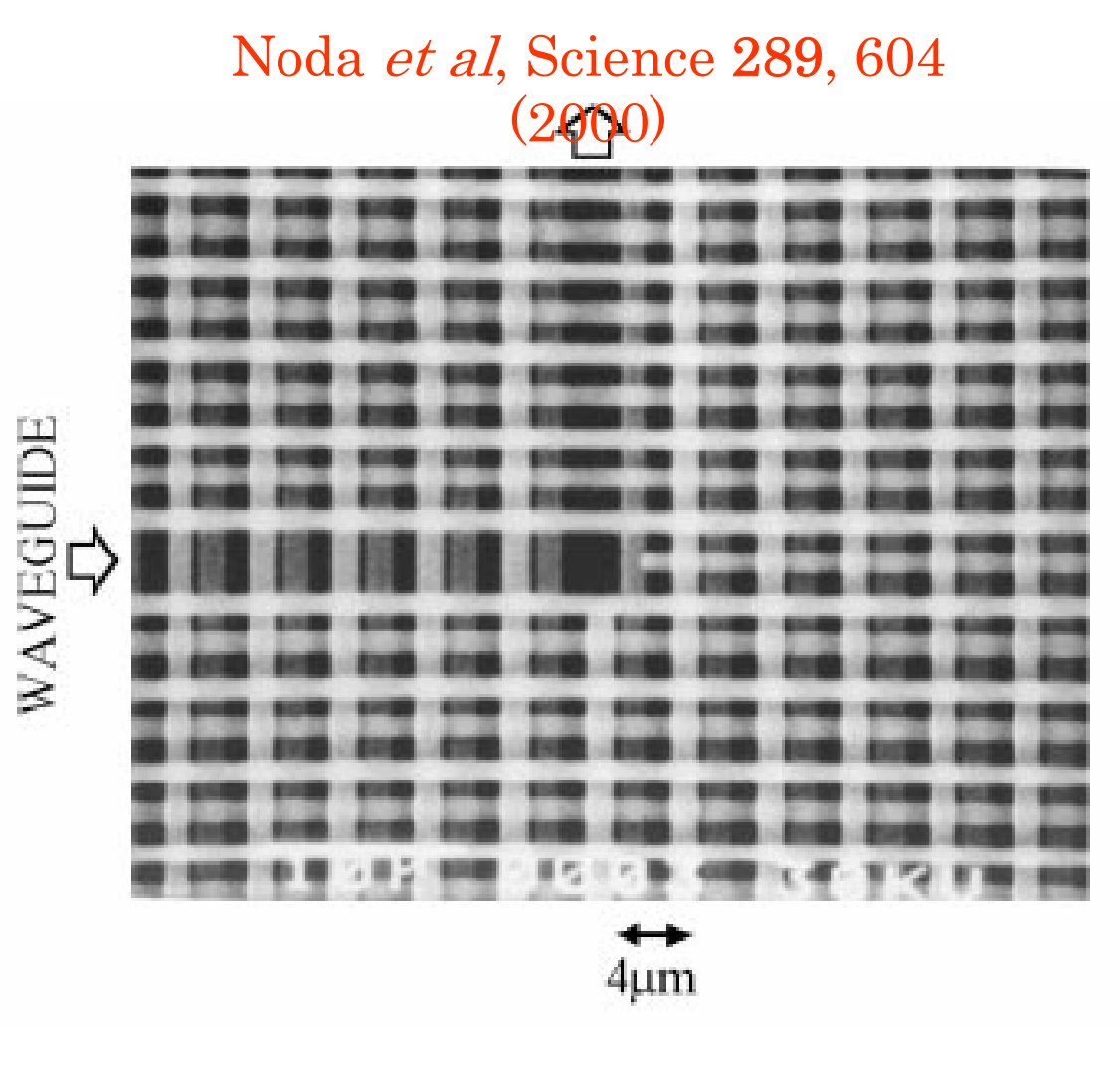

Noda et al, Science 289, 604

(2\&)

" Highly confined waveguides, waveguide bends, power splitter, add-dirop fillters, switches can be used in future ultrasmall optoelectronic integrated circuits. 
*arious applications of 1D, 2D, and 3D coupled-cavity structures were demonstrated

* The tight-binding approximation was successfully applied to the photonic structures

- The finite-difference-time-domain (FDTD) and the transfer matrix method (TMM) results agree well with our measurements

\section{ACKNOWLEDGEMENTS}

These works were supported by

$>$ Turkish Department of Defense Grant No. KOBRA-01, Thales JP8.04

$>$ NATO Grant No. SfP971970

$>$ National Science Foundation Grant No. INT-9820646

MORE INFORMATION

http://www.fen.bilkent.edu.tr/ bayindir

http://pbg.fen.bilkent.edu.tr 